\title{
A First and Second Order Moment Approach to Probabilistic Control Synthesis
}

\author{
Luis G. Crespo * \\ National Institute of Aerospace, Hampton, VA, 23666, USA \\ Sean P. Kenny ${ }^{\dagger}$ \\ Dynamic Systems and Control Branch, NASA LaRC, Hampton, VA, 23681
}

\begin{abstract}
This paper presents a robust control design methodology based on the estimation of the first two order moments of the random variables and processes that describe the controlled response. Synthesis is performed by solving an multi-objective optimization problem where stability and performance requirements in time- and frequency- domains are integrated. The use of the first two order moments allows for the efficient estimation of the cost function thus for a faster synthesis algorithm. While reliability requirements are taken into account by using bounds to failure probabilities, requirements related to undesirable variability are implemented by quantifying the concentration of the random outcome about a deterministic target. The Hammersley Sequence Sampling and the First- and Second-MomentSecond-Order approximations are used to estimate the moments, whose accuracy and associated computational complexity are compared numerically. Examples using output-feedback and full-state feedback with state estimation are used to demonstrate the ideas proposed.
\end{abstract}

*Staff Scientist, 144 Research Drive, Hampton VA 23666, AIAA Professional Member

$\dagger$ Aerospace Technologist, Dynamic Systems and Control Branch, NASA LaRC, Hampton VA 23681 


\section{Introduction}

Achieving balance between stability and performance in the presence of uncertainties is one of the fundamental challenges faced by control engineers. Trade-offs must be made to reach acceptable levels of stability and performance with adequate robustness to parameter uncertainty. These trade-offs are explicitly linked to the control engineer's choice of uncertainty model as well as how that model is exploited in the synthesis process. Usually, the assumed uncertainty model has a profound impact on the performance robustness of the closed-loop system.

Several uncertainty models, such as norm-bounded perturbations, interval analysis, fuzzy sets, and probabilistic methods ${ }^{1-3}$ are typically used. The most commonly used robust control methods ${ }^{4}$ are $\mu$-synthesis and $H$-infinity. In these methods, uncertainty is modeled with norm-bounded complex perturbations of arbitrary structure about a nominal plant. This treatment is used primarily because it leads to a tractable set of sufficient conditions for robust stability, making the approach computationally efficient. These methods are based on the most pessimistic value of performance among all possible ones, usually referred to as "worst-case". This worst-case performance is usually realized only by a single member of the uncertain model set and by a particular input signal. No information is provided regarding the likelihood that this worst-case will ever occur in practice. In addition, the intrinsic mathematical requirements of the approach usually lead to conservative models of uncertainty, over-conservative designs and complicated compensators.

Probabilistic uncertainty not only defines a set of plants where the actual dynamic system is assumed to reside but also associates a weight, the value of the probability density function, to each member of the set. In contrast to conventional robust control methods, this "additional dimension" allows the pursuit of robustly optimal solutions in the probabilistic sense. For instance, reliability-based design searches for solutions that minimize the probability of violating design requirements prescribed in terms of inequality constraints. Hence, reliability-based control design searches for the compensator that places as much probability as possible within the region where the design requirements are satisfied. Notice that this allows for the search of the compensator with the best robustness for a given control structure, e.g. the most robust PID controller. This can be achieved even though the violation of some the design requirements for some of the plants in the uncertainty set is unavoidable.

Synthesis approaches based on random searches ${ }^{5,6,14}$ and stochastic gradient algorithms ${ }^{8-10}$ 
have been applied to probabilistic robust control. In these studies, random sampling is the primary tool for assessing and pursuing acceptable levels of robustness in the control solution. On the other hand, asymptotic approximations ${ }^{11,12}$ for the estimation of failure probabilities have only been used as a control analysis tool. Reference, ${ }^{13}$ foundation of this study, integrates and extends some of these tools.

This paper is organized as follows. Section II presents basic concepts related to control and probabilistic uncertainty. Section III introduces reliability metrics for random variables and processes. Mean and variance based bounds to the reliability metrics are also derived therein. Robustness-based metrics for random variables and processes are introduced in Section IV. Section V presents the numerical methods used to estimate the above mentioned metrics. The control synthesis procedure is presented in Section VI, where specifics of both the reliability and the robustness-based formulations are examined. Two examples are presented in Section VII, where a satellite's attitude control problem and the disturbance rejection in a flexible beam are used to demonstrate the method. Finally, some conclusions are stated in Section VIII.

\section{System Dynamics}

Let $\mathbf{p}$ be a vector of random variables used to model the uncertain parameters of the system. In this study, $\mathbf{p}$ is prescribed a priori by the joint probability density function $(\mathrm{PDF}) f_{\mathbf{p}}(\mathbf{p})$ or equivalently by the cumulative distribution function $(\mathrm{CDF}) F_{\mathbf{p}}(\mathbf{p})^{\mathrm{a}}$. The set of values that $\mathbf{p}$ could take, called the support of $\mathbf{p}$, will be denoted as $\Delta_{\mathbf{p}}$.

Consider the probabilistic model $\mathcal{M}(\mathbf{p})$ of a Linear Time Invariant (LTI) system, where the dependence of the model on the uncertain parameters could be non-linear. The reader must notice however, that the developments presented herein do not require the system to be LTI. The propagation of $\Delta_{\mathbf{p}}$ through $\mathcal{M}$ leads to a set of uncertain plant models in which the physical system is assumed to reside. The probability distribution of a plant within this set is fully determined by $\mathcal{M}(\mathbf{p})$ and $f_{\mathbf{p}}(\mathbf{p})$. In a transfer function representation, we will refer to the uncertain plant as $G(\mathbf{p})$ and to the compensator as $K(\mathbf{k})$, where $\mathbf{k}$ is the vector of design parameters to be determined. Alternatively, a state space realization of $\mathcal{M}(\mathbf{p})$

\footnotetext{
${ }^{\text {a } I n ~ t h e s e ~ e x p r e s s i o n s, ~ t h e ~ s u b s c r i p t ~ r e f e r s ~ t o ~ t h e ~ s y m b o l ~ u s e d ~ f o r ~ t h e ~ r a n d o m ~ v a r i a b l e ~ w h i l e ~ t h e ~ v a l u e ~ i n ~}$ parenthesis refers to a particular realization.
} 
leads to

$$
\begin{aligned}
& \dot{\mathbf{x}}=\mathbf{A}(\mathbf{p}) \mathbf{x}+\mathbf{B}(\mathbf{p}) \mathbf{u}+\mathbf{F}(\mathbf{p}) \mathbf{z} \\
& \mathbf{y}=\mathbf{C}(\mathbf{p}) \mathbf{x}+\mathbf{D}(\mathbf{p}) \mathbf{u}+\mathbf{E}(\mathbf{p}) \mathbf{v}
\end{aligned}
$$

where $\mathbf{x}$ is the state, $\mathbf{u}$ is the control, $\mathbf{z}$ is process noise, $\mathbf{y}$ is the system output and $\mathbf{v}$ is sensor noise. The noise signals are commonly modeled as delta correlated Gaussian white noises satisfying $\mathrm{E}[\tilde{\mathbf{z}}]=\mathbf{0}$ and $\mathrm{E}\left[\tilde{\mathbf{z}}(t) \tilde{\mathbf{z}}^{T}(t+\kappa)\right]=\mathbf{S} \delta(\kappa)$, where $\tilde{\mathbf{z}}=\left[\mathbf{z}^{T}, \mathbf{v}^{T}\right]^{T}$ and $\mathbf{S}$ is a constant spectral density matrix. In what follows, the explicit dependence of the matrices in Equations (1-2) on $\mathbf{p}$ is omitted while $\mathbf{D}$ is assumed to be zero.

Important properties, typically used in control design, such as pole placement and the Separation Principle, do not hold due to the offset between the deterministic mathematical model and the actual dynamic system. The effects of parametric uncertainty on the Separation Principle are considered next. For the full-state feedback law $\mathbf{u}=-\mathbf{G} \hat{\mathbf{x}}$ and a full-order observer with gain $\mathbf{L}$ based on the expected plant $\mathrm{E}[\mathcal{M}(\mathbf{p})]$ (any other deterministic plant such as $\mathcal{M}(\mathrm{E}[\mathbf{p}])$ could be used instead), the closed-loop dynamics for full-state feedback is given by

$$
\begin{gathered}
\dot{\tilde{\mathbf{x}}}=\tilde{\mathbf{A}} \tilde{\mathbf{x}}+\tilde{\mathbf{B}} \tilde{\mathbf{z}} \\
\tilde{\mathbf{y}}=\tilde{\mathbf{C}} \tilde{\mathbf{x}}+\tilde{\mathbf{E}} \tilde{\mathbf{z}} \\
\tilde{\mathbf{A}}=\left[\begin{array}{c|c}
\mathbf{A}-\mathbf{B G} & \mathbf{B G} \\
\hline \mathbf{A}-\mathrm{E}[\mathbf{A}]+(\mathrm{E}[\mathbf{B}]-\mathbf{B}) \mathbf{G} & (\mathbf{B}-\mathrm{E}[\mathbf{B}]) \mathbf{G}+\mathrm{E}[\mathbf{A}]-\mathbf{L E}[\mathbf{C}] \\
+\mathbf{L}(\mathrm{E}[\mathbf{C}]-\mathbf{C}) & \\
\tilde{\mathbf{B}}=\left[\begin{array}{c|c}
\mathbf{F} & \mathbf{0} \\
\hline \mathbf{F} & -\mathbf{L E}
\end{array}\right]
\end{array}\right.
\end{gathered}
$$

where $\hat{\mathbf{x}}$ is the estimation of $\mathbf{x}, \tilde{\mathbf{x}}=\left[\mathbf{x}^{T}, \mathbf{e}^{T}\right]^{T}$ is the augmented state vector, $\mathbf{e}=\mathbf{x}-\hat{\mathbf{x}}$ is the estimation error, $\tilde{\mathbf{C}}=\left[\mathbf{C}^{T} \mid \mathbf{0}^{T}\right]^{T}$ and $\tilde{\mathbf{E}}=\left[\mathbf{0}^{T} \mid \mathbf{E}^{T}\right]^{T}$. The vector $\mathbf{k}$ is formed by the feedback gain $\mathbf{G}$ and the observer gain $\mathbf{L}$. Notice that the Separation Principle holds, i.e., 
$\tilde{\mathbf{A}}$ is upper triangular, if the deterministic plant used to generate the observer matches the physical dynamic system. Uncertainty in the plant makes the Separation Principle invalid. In addition, the random closed-loop poles do not occur at the locations selected for the full-state feedback, i.e. poles of the $\tilde{\mathbf{A}}_{1,1}$ subsystem, nor at the locations for the full-order observer, i.e. poles of the $\tilde{\mathbf{A}}_{2,2}$ subsystem.

\section{Reliability-Based Metrics}

The propagation of a fixed set of parameters of the plant through conventional control analysis tools leads to set of scalar quantities, e.g. closed loop poles, and a set of functions, e.g. step responses, and Bode plots. The propagation of probabilistic uncertainty through the same tools leads to random variables, e.g. random closed-loop poles, and random processes, e.g. the step responses become random processes parameterized by time, and the Bode plots become random processes parameterized by frequency. In this section we first introduce reliability metrics for random variables and processes. These metrics will be used to quantify the violation of the design requirements. Specific realizations corresponding to stability, time, and frequency requirements are then provided. In general, we will use $x$ and $x(h)$ to denote a random variable and a random process dependent on $\mathbf{p}$ through the plant model. For the random process $x(h), h$ refers to an arbitrary variable such as time or frequency.

\section{A. Random Variables}

We first introduce the concept of probability of failure. Let $x(\mathbf{p})$ be the random variable of interest. Let $x>\underline{x}$ be a design requirement. The event $x \leq \underline{x}$ will be referred to as failure. The corresponding failure set is given by $\mathcal{F}=\{x \mid x \in(-\infty, \underline{x}]\}$, where the failure boundary $\underline{x}$ is a deterministic quantity prescribed in advance. The admissible domain, namely $\mathcal{A}=$ $\{x \mid x \in(\underline{x}, \infty)\}$, is the complement of the failure domain. The same type of discrimination can be done in the parameter space $\mathbf{p}$ by using $x(\mathbf{p})$. The function $g(\mathbf{p}, \underline{x})=x(\mathbf{p})-\underline{x}$, called the limit state function, divides the parameter space in two parts, the domain leading to $\mathcal{A}$, i.e. $g(\mathbf{p}, \underline{x})>0$, and the domain leading to $\mathcal{F}$, i.e. $g(\mathbf{p}, \underline{x}) \leq 0$. Hence, $\mathcal{F}$ results from mapping the set $\left\{\mathbf{p} \in \Delta_{\mathbf{p}} \mid g(\mathbf{p}, \underline{x}) \leq 0\right\}$ through $x(\mathbf{p})$. In this case, the probability of failure 
$P_{f}$ is given by

$$
P_{f}=\mathrm{P}[x \leq \underline{x}]=\int_{x \leq \underline{x}} f_{x}(x) d x=\int_{g \leq 0} f_{\mathbf{p}}(\mathbf{p}) d \mathbf{p}
$$

Similar expressions can be derived if the design requirement is $x<\bar{x}$. These expressions describe reliability metrics for the random variable $x$ when a single constraint is present, i.e., $x>\underline{x}$ or $x<\bar{x}$. A reliability metric for the random variable $x$ having both constraints can be easily formed

$$
r_{x}(\underline{x}, \bar{x}) \triangleq \underline{r}_{x}(\underline{x})+\bar{r}_{x}(\bar{x})
$$

where

$$
\begin{aligned}
& \underline{r}_{x}(\underline{x}) \triangleq \mathrm{P}[x \leq \underline{x}]=F_{x}(\underline{x}) \\
& \bar{r}_{x}(\bar{x}) \triangleq \mathrm{P}[x>\bar{x}]=1-F_{x}(\bar{x})
\end{aligned}
$$

Notice that $\underline{r}_{x}(\underline{x})$ is equivalent to $P_{f}$ in Equation (5). We will refer to $\underline{x}$ and $\bar{x}$ as the boundaries of the failure domain $\mathcal{F}=\{x \mid x \in(-\infty, \underline{x}] \cup(\bar{x}, \infty)\}$. Notice that the under-bar and the over-bar refer to the bound from below and the bound from above of the admissible domain $\mathcal{A}=\{x \mid x \in[\underline{x}, \bar{x})\}$. This convention will be used for the remainder of the paper. Notice that the mapping of the corresponding limit state function through $x(\mathbf{p})$ leads to the failure boundary(s). Hence, there is a direct correspondence between $\mathcal{F}$ and $g$.

\section{B. Random Processes}

The random process $x(h)$ can be considered as the parameterization of the random variable $x$ by the deterministic variable $h$. In this paper $h \in[0, \infty]$ is assumed. The random process $x(h)$ is specified by the set of $\operatorname{CDFs}^{15} F_{x(h)}(x, h)$. For instance, the system output $y(t)$ is prescribed by $F_{y(t)}(y, t)$. The evaluation of the process at a particular $h$ value, say $h_{i}$, leads to the random variable $x\left(h_{i}\right)$ whose $\mathrm{CDF}$ is given by $F_{x}(x)=F_{x(h)}\left(x, h_{i}\right)$. In general, the support and the percentiles of $x(h)$ vary with $h$.

Let $x(h)>\underline{x}(h)$ for $h \in\left[h_{1}, h_{2}\right]$ and $x(h) \leq \bar{x}(h)$ for $h \in\left[h_{3}, h_{4}\right]$ be design requirements for the random process $x(h)$. In this paper, reliability metrics for processes are formulated by extending the ideas presented above. This is attained by integrating the reliability metric 
in Equation (6) for the random variable $x\left(h_{i}\right)$ in the $h$-interval of interest. In this context, a reliability metric for $x(h)$ is cast as

$$
r_{x(h)}(\underline{x}(h), \bar{x}(h)) \triangleq \underline{r}_{x(h)}(\underline{x}(h))+\bar{r}_{x(h)}(\bar{x}(h))
$$

where

$$
\begin{aligned}
& \underline{r}_{x(h)}(\underline{x}(h)) \triangleq \frac{1}{h_{2}-h_{1}} \int_{h_{1}}^{h_{2}} \mathrm{P}[x(h) \leq \underline{x}(h)] d h=\frac{1}{h_{2}-h_{1}} \int_{h_{1}}^{h_{2}} F_{x(h)}(\underline{x}(h), h) d h \\
& \bar{r}_{x(h)}(\bar{x}(h)) \triangleq \frac{1}{h_{4}-h_{3}} \int_{h_{3}}^{h_{4}} \mathrm{P}[x(h)>\bar{x}(h)] d h=\frac{1}{h_{4}-h_{3}} \int_{h_{3}}^{h_{4}} 1-F_{x(h)}(\bar{x}(h), h) d h
\end{aligned}
$$

are the costs of violating the lower and upper constraints respectively. These constraints, namely $\underline{x}(h)$ and $\bar{x}(h)$, will also be referred to as failure boundary functions. Notice that the failure domain

$$
\mathcal{F}=\left\{\bigcup_{h \in\left[h_{1}, h_{2}\right]}\{(x, h) \mid x \leq \underline{x}(h)\}\right\} \cup\left\{\bigcup_{h \in\left[h_{3}, h_{4}\right]}\{(x, h) \mid x \geq \bar{x}(h)\}\right\}
$$

is delimited by the failure boundaries. The reader shall realize that Equation (9) is a natural extension of Equation (6). If the process is contained within the set $\mathcal{A}$ the reliability metric $r_{x(h)}$ is zero, meaning that the inequality constraints are satisfied for all parameter values in $\Delta_{\mathbf{p}}$

\section{Realizations}

\section{Robust Stability}

A LTI system is robustly stable if all its poles are in the open left half of the complex plane for all possible values of the random parameters. A reliability assessment of stability is given by

$$
\mathrm{P}\left[\bigcup_{i=1}^{v}\left(\Re\left[s_{i}\right]>0\right)\right]=\epsilon
$$

where $s_{i}$ with $i=1,2, \ldots v$ is a random pole, $\Re[\cdot]$ is the real part operator and $\epsilon$ is the resulting probability of instability. Robust stability is attained if $\epsilon=0$. Stability can also 
be cast via

$$
\lambda \triangleq \max \left\{\Re\left[s_{1}\right], \Re\left[s_{2}\right], \ldots, \Re\left[s_{v}\right]\right\}
$$

In terms of $\lambda$, the probability of instability is given by $\bar{r}_{\lambda}(0)$. Robust stability is attained if $\bar{r}_{\lambda}(0)=0$. Several comments are now pertinent. Reaching robust stability may not be feasible for the given support $\Delta_{\mathbf{p}}$ (even if it is bounded) and the assumed control structure $K(\mathbf{k})$. Notice also that the acceptance of a small non-zero probability of instability could be desirable from the performance point of view. For instance, allowing the right low-probability tail of $f_{\lambda}(\lambda)$ to lie on the open right half of the complex plane may yield a significant enhancement in the performance of the plants associated with the high probability portions of the PDF. Rather than advocating for the acceptance of the risk that this practice implies, we would like to highlight that the trade-off between robustness and performance can be studied by allowing small values of $\epsilon$.

\section{Time-Domain}

Quite frequently performance requirements are prescribed in terms of time-domain specifications. The propagation of $f_{\mathbf{p}}(\mathbf{p})$ through the system dynamics leads to random processes for the time responses. Denote by $x(t)$ an arbitrary random process with CDF $F_{x(t)}(x, t)$. Such process is parameterized by $\mathbf{p}$, time $t$, and the compensator design variable $\mathbf{k}$. The dependence of $x(t)$ on $\mathbf{k}$ has been omitted for the sake of simplifying the notation. Reliability metrics for relevant processes can be cast using Equation (9). For instance, while settling time and overshoot requirements are integrated using $r_{y(t)}(\underline{y}(t), \bar{y}(t))$, the control saturation requirement $|u|<u_{\max }$ leads to $r_{u(t)}\left(-u_{\max }, u_{\max }\right)$.

A reliability metric for assessing the effects of noise on the uncertain plant is formulated next. The state covariance matrix, defined as $\mathbf{Q}(t)=\mathrm{E}\left[\tilde{\mathbf{x}}(t) \tilde{\mathbf{x}}^{T}(t)\right]$, is given by the solution to the covariance equation

$$
\dot{\mathbf{Q}}=\tilde{\mathbf{A}} \mathbf{Q}+\mathbf{Q} \tilde{\mathbf{A}}^{T}+\tilde{\mathbf{B}} \mathbf{S} \tilde{\mathbf{B}}^{T}
$$

subject to $\mathbf{Q}(0)=\mathbf{Q}_{\mathbf{0}}$. The output covariance, defined as $\mathrm{E}\left[\tilde{\mathbf{y}}(t) \tilde{\mathbf{y}}^{T}(t)\right]$, reaches the steady- 
state Root Mean Square (RMS) value

$$
\tilde{\mathbf{y}}_{r m s}=\lim _{t \rightarrow \infty}\left(\operatorname{diag}\left[\tilde{\mathbf{C}} \mathbf{Q}(t) \tilde{\mathbf{C}}^{T}\right]\right)^{1 / 2}
$$

Notice that uncertainty in $\mathbf{p}$ makes $\tilde{\mathbf{y}}_{r m s}$ a random vector. If $y_{r m s}$ is a component of $\tilde{\mathbf{y}}_{r m s}$, a reliability metric that penalizes the violation $y_{r m s}>\bar{y}_{r m s}$ is given by $\bar{r}_{y_{r m s}}\left(\bar{y}_{r m s}\right)$.

\section{Frequency-Domain}

The propagation of $f_{\mathbf{p}}(\mathbf{p})$ through the system dynamics onto the frequency domain leads to random processes of the form $x(\omega)$, fully specified by $F_{x(\omega)}(x, \omega)$. Here, $x(\omega)$ is any real frequency dependent metric of the feedback loop, e.g. Bode magnitude. This random process is parameterized by $\mathbf{p}$, frequency $\omega$, and the compensator design variable $\mathbf{k}$. A reliability metric for $x(\omega)$ is $r_{x(\omega)}(\bar{x}(\omega), \underline{x}(\omega))$. For instance, conventional control requirements ${ }^{16}$ for disturbance rejection, noise attenuation and reference tracking can be cast in terms of the loop

transfer function $q(\omega) \triangleq|G K|$. Low frequency requirements can be cast using $\underline{r}_{q(\omega)}(\underline{q}(\omega))$ with $\underline{q}(\omega)=1$ and high frequency requirements with $\bar{r}_{q(\omega)}(\bar{q}(\omega))$ for which $\bar{q}(\omega)$ has a proper roll off.

\section{Reliability Bounds}

The following lemma defines bounds for the reliability metrics in terms of the first two order moments.

Lemma 1. Let $\mathrm{E}[\cdot]$ and $\mathrm{V}[\cdot]$ denote the expected value and variance operators.

$$
\begin{aligned}
& \underline{r}_{x}(\underline{x}) \leq \frac{\mathrm{V}[x]}{(\mathrm{E}[x]-x)^{2}} \text { if } \underline{x}<\mathrm{E}[x] \\
& \bar{r}_{x}(\bar{x}) \leq \frac{\mathrm{V}[x]}{(\bar{x}-\mathrm{E}[x])^{2}} \text { if } \bar{x}>\mathrm{E}[x]
\end{aligned}
$$

Proof. If $\epsilon>0$

$$
\mathrm{V}[x] \geq \int_{|x-\mathrm{E}[x]|>\epsilon}(x-\mathrm{E}[x])^{2} f_{x}(x) d x \geq \epsilon^{2} \mathrm{P}[|x-\mathrm{E}[x]|>\epsilon] \geq \epsilon^{2} \mathrm{P}[x<\mathrm{E}[x]-\epsilon]
$$

We obtain Equation (15) using $\underline{x}=\mathrm{E}[x]-\epsilon$. Equation (16) is derived following the same lines. 
Notice that these bounds apply to any PDF of $x$. Since the bounds given in Equations (15-16) are solely dependent on the first two order moments they can be estimated more efficiently than exact failure probabilities. Notice however that the use of bounds instead of reliability metric introduces conservatism into the solution.

$$
b_{x}(\underline{x}, \bar{x}) \triangleq \underline{b}_{x}(\underline{x})+\bar{b}_{x}(\bar{x})
$$

where

$$
\begin{aligned}
& \underline{b}_{x}(\underline{x}) \triangleq \begin{cases}\frac{\mathrm{V}[x]}{(\mathrm{E}[x]-\underline{x})^{2}} & \text { if } \underline{x}<\mathrm{E}[x] \\
\infty & \text { otherwise }\end{cases} \\
& \bar{b}_{x}(\bar{x}) \triangleq \begin{cases}\frac{\mathrm{V}[x]}{(\bar{x}-\mathrm{E}[x])^{2}} & \text { if } \bar{x}>\mathrm{E}[x] \\
\infty & \text { otherwise }\end{cases}
\end{aligned}
$$

As before, the under-bar and over-bar in $b$ refer to the way in which the failure event is defined. Notice that $r_{x} \leq b_{x}, \underline{r}_{x}(\underline{x}) \leq \underline{b}_{x}(\underline{x})$, and $\bar{r}_{x}(\bar{x}) \leq \bar{b}_{x}(\bar{x})$. The same idea, extended to random processes, leads to

$$
b_{x(h)}(\underline{x}(h), \bar{x}(h)) \triangleq \underline{b}_{x(h)}(\underline{x}(\cdot))+\bar{b}_{x(h)}(\bar{x}(h))
$$

where

$$
\begin{aligned}
& \underline{b}_{x(h)}(\underline{x}(h)) \triangleq \begin{cases}\frac{1}{h_{2}-h_{1}} \int_{h_{1}}^{h_{2}} \underline{b}_{x}(\underline{x}(h)) d h & \text { if } \underline{x}(h)<\mathrm{E}[x(h)] \forall h \in\left[h_{1}, h_{2}\right] \\
\infty & \text { otherwise }\end{cases} \\
& \bar{b}_{x(h)}(\bar{x}(h)) \triangleq \begin{cases}\frac{1}{h_{4}-h_{3}} \int_{h_{3}}^{h_{4}} \bar{b}_{x}(\bar{x}(h)) d h & \text { if } \bar{x}(h)>\mathrm{E}[x(h)] \forall h \in\left[h_{3}, h_{4}\right] \\
\infty & \text { otherwise }\end{cases}
\end{aligned}
$$

As before, $r_{x(h)} \leq b_{x(h)}, \underline{r}_{x(h)}(\underline{x}(h)) \leq \underline{b}_{x(h)}(\underline{x}(h))$ and $\bar{r}_{x(h)}(\bar{x}(h)) \leq \bar{b}_{x(h)}(\bar{x}(h))$.

\section{Robustness-Based Metrics}

Some performance requirements might not be properly captured in a conventional reliability formulation since they focus on the bulk portion of the PDF. Throughout this paper the term robustness is used to describe the design characteristic that evaluates the performance degradation from an ideal deterministic behavior caused by uncertainty. Robustness 
metrics, that quantify such a characteristic, are presented next. For random variables, the index

$$
\tau_{x}(\hat{x})=\int_{\Delta_{x}}(\xi-\hat{x})^{2} f_{x}(\xi) d \xi=\mathrm{V}[x]+\mathrm{E}[x](\mathrm{E}[x]-2 \hat{x})+\hat{x}^{2}
$$

is a measure of the concentration of $f_{x}(x)$ about the deterministic target value $\hat{x}$. In the ideal case when $f_{x}(x)=\delta(x-\hat{x})$, we obtain $\tau_{x}(\hat{x})=0$. For random processes, the index

$$
\tau_{x(h)}(\hat{x}(\cdot))=\frac{1}{h_{6}-h_{5}} \int_{h_{5}}^{h_{6}} \tau_{x}(\hat{x}(h)) d h
$$

is a measure of the concentration of the process $x(h)$ about the deterministic target function $\hat{x}(h)$ in $h \in\left[h_{5}, h_{6}\right]$. The reader must notice that the evaluation of the above expressions only requires of the first two order moments of the process.

Robustness-based metrics parallel to the ones provided in Section C can easily be posed. For instance, if $y_{r m s}$ is the RMS steady state value of an error signal, the index $\tau_{y r m s}(0)$ quantifies the offset between the target behavior $\hat{y}_{r m s}=0$ and the random variable $y_{r m s}$. Likewise, the metric $\tau_{u(t)}(0)$ quantifies the offset between the random process $u(t)$ and the target $\hat{u}=0$, for which no actuation is required.

\section{Numerical Estimation}

Since the estimation of the metrics for random processes are a natural extension of the methods for random variables, only the estimation of the statistics of $x$ is addressed here. The extension to processes is as follows. For the random process $x(h)$, create a uniform sample of $e$ points $h_{1}, h_{2}, \ldots, h_{e}$ in the $h$-domain. We will refer to these samples as the $e$ $h$-samples. Statistics for the resulting $e$ random variables $x_{i}=x\left(h_{i}\right), i=1,2 \ldots e$ are then used to estimate the pertinent integrals, i.e. Equations (10-11), (21-22) and (24).

\section{A. Mean and Variances}

The reliability bounds and the robustness metrics introduced above depend exclusively on mean and variances. In this paper, Sampling and the First and Second Moment Second Order approximations are used for their estimation. These methods are briefly introduced next. 


\section{Sampling}

Unbiased estimators for the mean and variance of the random variable $x$ are

$$
\begin{aligned}
& \mathrm{E}[x] \approx \frac{1}{n} \sum_{i=1}^{n} x_{i} \\
& \mathrm{~V}[x] \approx \frac{1}{n-1} \sum_{i=1}^{n}\left(x_{i}-\mathrm{E}[x]\right)^{2}
\end{aligned}
$$

where $x_{i}=x\left(\mathbf{p}_{i}\right)$ is the $i t h$ sample. The $\mathbf{p}_{i}$ sample of $f_{\mathbf{p}}(\mathbf{p})$ can be generated by any sampling technique.

HSS generates representative deterministic samples of $f_{\mathbf{p}}(\mathbf{p})$. The error of approximating an integral by a finite number of samples of the integrand, depends on the uniformity of the points used to generate the samples rather than on their randomness. This fact has motivated the development of deterministic sampling techniques such as HSS. While conventional Monte Carlo Sampling (MCS) is based on the generation of random points on the unit hypercube, HSS is based on the generation of an evenly distributed set points. The reader can refer to ${ }^{13}$ for details on HSS implementation.

HSS requires far fewer samples ${ }^{18}$ than $\mathrm{MCS}^{5-7,14}$ for a given confidence level. Improvements in the convergence rate of the estimated first two order moments by a factor of three to one hundred ${ }^{19}$ have been reported. In addition, the estimated value of the failure probability based on HSS is deterministic. In contrast, MCS leads to random estimates unless an infinite number of samples is used. This is especially noticeable if $n$ is small. The random character of the estimation can only be mitigated by increasing the number of samples, which incidentally increases the computational demands of algorithms based on MCS. Therefore, HSS not only leads to more accurate estimations than MCS for a given number of samples but also eliminates the random character of the results.

\section{First and Second Moment Second Order Approximations (FSMSO)}

These approximations result from calculating the first and second moment of a second order Taylor expansion of $x(\mathbf{p})$ about $\mathrm{E}[\mathbf{p}]$. Let $\mathbf{p} \in \mathbb{R}^{m}$, and $\mathbf{p}_{i}$ be the ith component of $\mathbf{p}$. If the 
components of $\mathbf{p}$ are independent random variables, the resulting approximations are

$$
\begin{aligned}
\mathrm{E}[x] & \approx x+\frac{1}{2} \sum_{i=1}^{m} \frac{\partial^{2} x}{\partial \mathbf{p}_{(i)}^{2}} \mathrm{~V}\left[\mathbf{p}_{(i)}\right] \\
\mathrm{V}[x] & \approx \sum_{i=1}^{m}\left[\left(\frac{\partial x}{\partial \mathbf{p}_{(i)}}\right)^{2} \mathrm{~V}\left[\mathbf{p}_{(i)}\right]+\left(\frac{\partial x}{\partial \mathbf{p}_{(i)}}\right)\left(\frac{\partial^{2} x}{\partial \mathbf{p}_{(i)}^{2}}\right) \mathrm{T}\left[\mathbf{p}_{(i)}\right]+\frac{1}{4}\left(\frac{\partial^{2} x}{\partial \mathbf{p}_{(i)}^{2}}\right)^{2}\left(\mathrm{~F}\left[\mathbf{p}_{(i)}\right]-\mathrm{V}\left[\mathbf{p}_{(i)}\right]^{2}\right)\right] \\
& +\sum_{i=1}^{m} \sum_{j \neq i}^{m} \frac{1}{2}\left(\frac{\partial^{2} x}{\partial \mathbf{p}_{(i)} \partial \mathbf{p}_{(j)}}\right)^{2} \mathrm{~V}\left[\mathbf{p}_{(i)}\right] \mathrm{V}\left[\mathbf{p}_{(j)}\right]
\end{aligned}
$$

where the functions and derivatives are evaluated at $\mathrm{E}[\mathbf{p}], \mathrm{T}[\cdot]$ is the third central moment operator and $\mathrm{F}[\cdot]$ is the fourth.

In general, reliability metrics cannot be evaluated exactly since they involve the evaluation of complicated integrals, usually multi-dimensional, over complex domains. The estimation of failure probabilities, basic component of the reliability metrics, can be done using sampling or asymptotic approximations. The reader can refer to ${ }^{13}$ for details on their estimation.

\section{Control Synthesis}

\section{A. Reliability-based}

The formulation of the control design problem from a reliability perspective is as follows. For a given plant model, compensator structure, uncertainty model, and a set of design requirements prescribed via inequality constraints; one would like to find the compensator parameters for which the resulting probability of violating the design requirements is minimized. Notice that this refers to the excursion of the outcomes into the failure domains.

\section{Robustness Considerations}

The reliability metrics in Equations (6-9) are usually applied using a fixed failure set $\mathcal{F}$. In this form, a reliability analysis cannot assess the system's performance in the regions where the design requirements are satisfied, i.e. the intersection of the admissible domains associated with all the design requirements. Since the portion of the random outcome lying in the admissible domain $\mathcal{A}$ might end up being substantially larger than the portion lying in the failure domain $\mathcal{F}$, a reliability-based approach with fixed failure boundaries does not 
have control over the bulk portion of the PDF, which is the portion that dictates the most likely performance.

We now introduce the concept of a shapeable failure set $\mathcal{F}$ with an example. Let $x(\mathbf{k})$ be the stationary RMS value of an error signal. One would like to find $\mathbf{k}$ such that $x$ is as close as possible to zero. Uncertainty in the plant makes $x$ a random variable. Let $\bar{x}$ be the failure boundary associated with the design requirement $x<\bar{x}$, i.e. a fixed failure set is $\mathcal{F}=\{x \mid x \in[\bar{x}, \infty)\}$. The minimization of $\bar{r}_{x}(\bar{x})$ leads to reliability optimal compensators. Suppose there exist multiple designs leading to $\bar{r}_{x}(\bar{x})=0$. These designs however differ in how well the resulting PDF of $x$ spreads over the admissible domain $\mathcal{A}=\{x \mid x \in[0, \bar{x})\}$. The concentration of $f_{x}(x)$ about zero is an indicator of the robust performance. Say, for example, that $\mathbf{k}_{1}$ leads to $\bar{r}_{x}(\bar{x} / 2)=0$ and $\mathbf{k}_{2}$ leads to $\bar{r}_{x}(\bar{x})=0$. Since neither of these two designs violate the design requirement, a reliability analysis cannot establish that the compensator with parameters $\mathbf{k}_{1}$ has a better robust performance than the one which uses $\mathbf{k}_{2}$.

By minimizing the reliability metrics and simultaneously shrinking the admissible domain, the whole random variable/process can be concentrated about regions with an improved system performance. This is attained by parameterizing the failure boundaries of $\mathcal{F}$ as well as a penalizing function $\gamma_{x}$ with an additional design variable, namely e. The basic idea is to solve an optimization problem for the design variable $\mathbf{d}=[\mathbf{k}, \mathbf{e}]$ such that a twofold objective is pursued: a reliability metric is minimized while the size of $\mathcal{A}$ is reduced. For the RMS example above, the minimization of $J=\bar{r}_{x}(\mathbf{e})+\gamma_{x}$ where $\gamma_{x}=\mathbf{e}, \mathbf{d}=[\mathbf{k}, \mathbf{e}]$ and $\mathbf{e} \in[0, \bar{x}]$, leads to the desired solution. This setting implies $\mathcal{F}=\{x \mid x \in[\mathbf{e}, \infty)\}$ and $\mathcal{A}=\{x \mid x \in[0, \mathbf{e})\}$. See Figure 1. Notice that the value of $J$ for $\mathbf{k}_{1}$ is less than the one for $\mathbf{k}_{2}$ if $\mathbf{e} \in[\bar{x} / 2, \bar{x})$. In general, we will refer to the augmented reliability metric as the sum of a reliability metric from Section III and a penalizing term. Augmented reliability metrics for the random variable $x$ and the random process $x(h)$ take the form $r_{x}(\underline{x}(\mathbf{e}), \bar{x}(\mathbf{e}))+\gamma_{x}(\mathbf{e})$ and $r_{x(h)}(\underline{x}(h, \mathbf{e}), \bar{x}(h, \mathbf{e}))+\gamma_{x(h)}(\mathbf{e})$ The penalizing functions $\gamma_{x}(\mathbf{e})$ and $\gamma_{x(h)}(\mathbf{e})$ must be proportional to the size of the admissible domain $\mathcal{A}$. In addition, they must be built such that the minimization of the augmented metric does not lead to unacceptable solutions, e.g. $r_{x}=1$ and $\gamma_{x}=0$. If $r_{x}<\epsilon$ is required, use a monotonically increasing function satisfying $\gamma \in[0, \epsilon]$. In the RMS example above, this is attained by minimizing an augmented reliability metric with $\gamma_{x}(\mathbf{e})=\epsilon \mathbf{e} / \bar{x}$ for $\mathbf{e} \in[0, \bar{x}]$. 


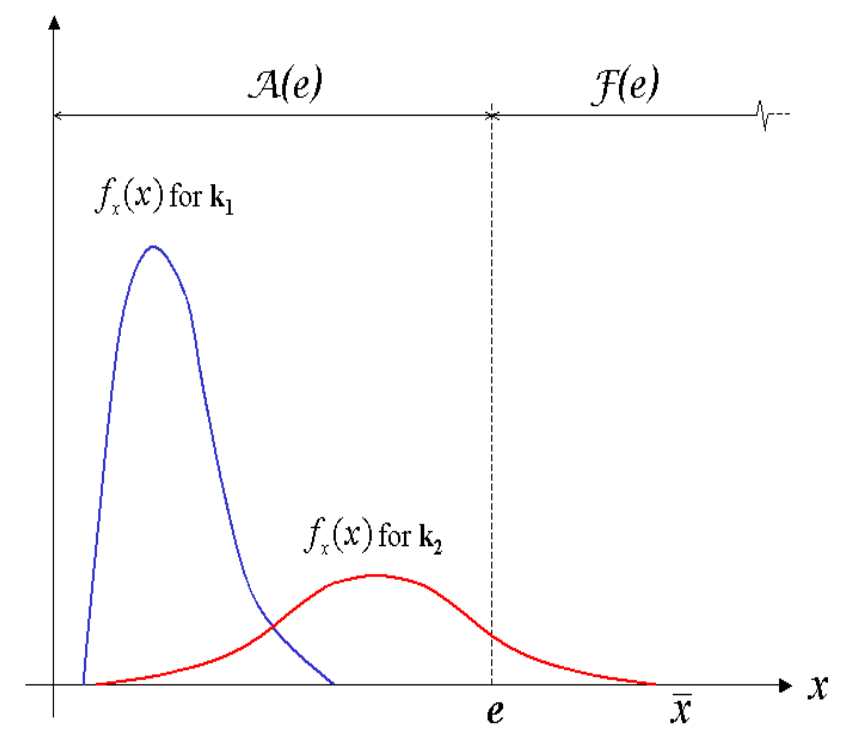

Figure 1. Robust performance concepts and shapeable failure domains

\section{B. Robustness-based}

The formulation of the control design problem from a robustness perspective is as follows. For a given plant model, compensator structure, and uncertainty model; one would like to find the compensator parameters for which the resulting random outcome is as concentrated as possible to a target deterministic behavior. While the deterministic target for a random variable is a scalar, the target for a random process is a function, e.g., $\hat{u}(t)=0$ is the target function for the control.

\section{Synthesis Procedure}

A step-by-step procedure to control synthesis is presented next.

1. Determine the plant model and the control structure. First principles and classical deterministic approaches to compensator design can be used. Identify the set of parameters that have a strong impact on the plant model. Use sensitivity information and engineering judgment to select the set of uncertain parameters $\mathbf{p}$. At this stage, the parametric plant model, i.e. $G(\mathbf{p})$, and the control structure, i.e. $K(\mathbf{k})$, must be fully determined. 
2. Generate the probabilistic parameter model $f_{\mathbf{p}}(\mathbf{p})$. Use engineering judgment and experimental data if available.

3. Cast the design requirements, either reliability or robustness based, in terms of the metrics introduced above. Use Equations (6,9) for the reliability metrics and Equations $(23,24)$ for the robustness metrics. Recall that while each reliability requirement requires setting a failure domain $\mathcal{F}$, each robustness requirement requires setting a target behavior. Use these metrics to compose the cost vector $\mathbf{c}$, which is the vector of objectives for multi-objective optimization.

4. Let $\mathbf{d}$ be the design variable. For robustness-based metrics and reliability-based metrics with fixed failure domains, $\mathbf{d}=\mathbf{k}$. Reliability-metrics with shapeable failure domains lead to $\mathbf{d}=\left[\mathbf{k}^{T}, \mathbf{e}^{T}\right]^{T}$. Build a penalizing function $\gamma(\mathbf{e})$ for these terms. Update the components of the cost vector $\mathbf{c}$ by adding the penalizing functions and parameterizing the failure boundaries.

5. Solve the single objective optimization problem

$$
\mathbf{d}^{*}=\operatorname{argmin}\left\{\mathbf{c}^{\mathrm{T}} \mathbf{w}\right\}
$$

where $\mathbf{w}$ is composed of non-negative weights. Each cost function evaluation used in the search for the optimal design $\mathbf{d}^{*}$ requires a probabilistic analysis. This analysis is done by calculating the metrics contained in c. Either sampling or FSMSO can be used to estimate the mean and variance based metrics introduced above. This task requires forming the closed-loop Equations (3-4) and performing typical control studies such as finding closed loop poles, time responses and Bode plots.

The cost vector c can be formed by combinations of reliability-based metrics, reliability bounds and/or robustness-based metrics. The corresponding implications are explored in the examples.

\section{Optimization under Uncertainty}

Let $J(\mathbf{p}, \mathbf{d})=\mathbf{c}^{T} \mathbf{w}$ denote the cost function of the optimization problem. If reliability metrics are used in in $\mathbf{c}$, the cost function might not only have plateaus, i.e., there could exist a design $\mathbf{d}$ and a non-zero perturbation $\delta$ such that $J(\mathbf{p}, \mathbf{d})=J(\mathbf{p}, \mathbf{d}+\delta)$, but might 
also have a discontinuous gradient. The use of sampling in the estimation of probabilities makes the cost function piecewise constant. Let $J_{e}(\mathbf{p}, \mathbf{d})$ be an estimation of the actual cost $J(\mathbf{p}, \mathbf{d})$. For any design $\mathbf{d}$ and regardless of the number of samples, there always exists a perturbation $\delta$ such that $J_{e}(\mathbf{p}, \mathbf{d})=J_{e}(\mathbf{p}, \mathbf{d}+\delta)$. This situation is aggravated, i.e. bigger perturbations can be found, when a smaller number of samples is used or when $P_{f}$ is close to zero or one.

Mean and variances can be estimated via HSS or FSMSO. Their estimation via sampling does not exhibit the numerical problems mentioned above. Among the sampling techniques, it was found that HSS suites very well the need for an accurate and efficient estimation. ${ }^{18}$ Estimation via FSMSO is considerably faster than sampling but less accurate in general. This is because, the Taylor expansion might become a poor approximation of the actual function when $f_{\mathbf{p}}(\mathbf{p})$ is not concentrated about $\mathrm{E}[\mathbf{p}]$. The methods used for the estimation of $J_{e}$ must be taken into account when selecting an optimization algorithm. In this paper, we use the Nelder Mead Simplex algorithm, which is a local non-gradient based search method.

\section{Numerical Examples}

The synthesis procedure of Section $\mathrm{C}$ is applied herein to two examples. A textbook satellite attitude control problem is considered first. Then, the active control of a flexible beam subject to disturbances is considered. The following parameter values are assumed in both problems. If $\mathbf{p} \in \mathbb{R}^{m}$, and sampling is used, a coarse probabilistic analysis is performed using $n=75 m \mathbf{p}$-samples and $e=90 h$-samples. If the resulting assessment is satisfactory, a new analysis is performed using $n=500 \mathrm{~m} \mathbf{p}$-samples and $e=180 h$-samples. This two-step analysis is carried out to adjust the fidelity of the estimation according to the characteristics of the compensator. For the sake of comparison, the examples also present the analysis of deterministic versions of the problems for which $E[\mathbf{p}]$ is used as parameter values. Such problems and their solutions will be referred to as the nominal ones.

\section{A. Satellite Attitude Control}

Accurate satellite pointing in the presence of large thermal gradients and mass losses for uncertain initial conditions is desired. A simple rotational model of two bodies connected 
with a flexible boom leads to

$$
\begin{aligned}
& J_{1} \ddot{\theta}_{1}+b\left(\dot{\theta}_{1}-\dot{\theta}_{2}\right)+k\left(\theta_{1}-\theta_{2}\right)=u \\
& J_{2} \ddot{\theta}_{2}+b\left(\dot{\theta}_{2}-\dot{\theta}_{1}\right)+k\left(\theta_{2}-\theta_{1}\right)=0
\end{aligned}
$$

where $\theta_{1}$ and $\theta_{2}$ are the deflection angles, $J_{1}$ and $J_{2}$ are moments of inertia, $k$ is the equivalent stiffness, $b=a \sqrt{k / 10}$ is the equivalent damping coefficient and $u$ is an applied torque. The variable $a$ is used to model the changes in damping caused by thermal variations. Assume that $J_{2}=0.1$ since mass losses only affect $J_{1}$. The non-collocated sensor-actuator pair resulting from using $y=\theta_{2}$ leads to the SISO system

$$
G(\mathbf{p})=\frac{k+b s}{J_{1} J_{2} s^{4}+b\left(J_{1}+J_{2}\right) s^{3}+\left(J_{1}+J_{2}\right) k s^{2}}
$$

Variations in the operating conditions and ignorance on the initial conditions are modeled using $\mathbf{p}=\left[J_{1}, a, k, \theta_{10}, \dot{\theta}_{10}, \theta_{20}, \dot{\theta}_{20}\right]^{T}$, where $\theta_{10}=\theta_{1}(0)$ and $\theta_{20}=\theta_{2}(0)$. The following output-feedback control structure is assumed

$$
K(\mathbf{k})=\frac{k_{1}+k_{2} s+k_{3} s^{2}+k_{4} s^{3}}{k_{5}+k_{6} s+k_{7} s^{2}+k_{8} s^{3}}
$$

The joint PDF that describes the uncertainty in $\mathbf{p}$ is given by the independent random variables listed in Table 1, where $U$ and $B$ refer to Uniform and Beta distributions. Notice that the Beta distribution has four independent parameters, two of them are the conventional arguments and the other two are the support bounds.

\section{Nominal Compensator}

A baseline compensator for the nominal plant is designed by standard pole placement techniques such that the resulting closed-loop system is robustly stable for the uncertainty set defined above. This was achieved by pursuing large stability margins. This practice results in a compensator with parameters $\mathbf{k}_{1}=10^{6}[0.0108,-0.3271,0.1192,0.0092,1.8835,2.1305$, $2.2276,0.9308]^{T}$. The analysis of the nominal compensator using $f_{\mathbf{p}}(\mathbf{p})$ indicates that the closed-loop system is robustly stable as intended, i.e. $\bar{r}_{\lambda}(0)=0$, but the time responses are unsatisfactory. The CDF of $\lambda$ as well as the time evolutions of the output and the control 
Table 1. Uncertainty model.

\begin{tabular}{|c|c|c|}
\hline$J_{1}$ & $\Delta_{J_{1}}=[0.8,1]$ & $f_{J_{1}}\left(J_{1}\right)=U(0.8,1)$ \\
\hline$a$ & $\Delta_{a}=[0.03,0.2]$ & $f_{a}(a)=B(0.3,0.2)$ \\
\hline$k$ & $\Delta_{k}=[0.09,0.4]$ & $f_{k}(k)=B(5,5)$ \\
\hline$\theta_{10}$ & $\Delta_{\theta_{10}}=[-\pi / 2, \pi / 2]$ & $f_{\theta_{10}}\left(\theta_{10}\right)=B(5.2,5.2)$ \\
\hline$\dot{\theta}_{10}$ & $\Delta_{\dot{\theta}_{10}}=[-15,15]$ & $f_{\dot{\theta}_{10}}\left(\dot{\theta}_{10}\right)=B(2.5,2.5)$ \\
\hline$\theta_{20}$ & $\Delta_{\theta_{20}}=[-\pi / 2, \pi / 2]$ & $f_{\theta_{20}}\left(\theta_{20}\right)=B(5.2,5.2)$ \\
\hline$\dot{\theta}_{20}$ & $\Delta_{\dot{\theta}_{20}}=[-15,15]$ & $f_{\dot{\theta}_{20}}\left(\dot{\theta}_{20}\right)=B(2.5,2.5)$ \\
\hline
\end{tabular}

signals are shown in Figures 2-4. The sudden variation in the slope of the CDF of $\lambda$ in Figure 2 is the result of a change in the closed-loop pole that determines $\lambda$. The considerable disparity between $\lambda(\mathrm{E}[\mathbf{p}])$ and $\mathrm{E}[\lambda(\mathbf{p})]$ shows that the nominal problem is not a meaningful representative of the probabilistic behavior. Figures 3 and 4 show the time evolution of the random signals by indicating the 1, 10, 20, 30, 40, 50,60, 70, 80, 90 and 99 percentiles. In Figures 3 and 4, the percentiles and the nominal functions are shown. Dotted lines are used to indicate the failure boundaries, specified in the next section. It is interesting to see how the PDFs expand, e.g. Figure 4 at 2.5 and 8 seconds, and contract, e.g. Figure 4 at 4 and 16 seconds, in a oscillatory manner. This information can be used to determine the time periods when the effects of uncertainty are more noticeable.

\section{Mean and Variance based compensator}

If only means and variances are used, reliability and robustness metrics can be combined to form $\mathbf{c}=\left[\bar{b}_{\lambda}(0), \tau_{y(t)}(1), \tau_{u(t)}(0), \underline{b}_{q(\omega)}(1), \bar{b}_{q(\omega)}(0.75 / \omega)\right]^{T}$. Ranges provided before will be used with the exception of $\omega \in\left[5,10^{2}\right]$ for $\bar{b}$. The cost vector can be estimated via sampling or the FSMSM.

HSS leads to $\mathbf{k}_{3}=10^{5}[1.1207,0.2307,1.5235,0.1713,0.001,0.0692,1.2294,1.069]^{T}$ and $\mathbf{c}=$ $\left[8.6 \times 10^{-5}, 0.0057,0.0129,1.94 \times 10^{-4}, 6.189 \times 10^{-5}\right]^{T}$ for which the weighting vector $\mathbf{w}=$ $[500,1,4,1,1]^{T}$ was used. A probabilistic analysis of this compensator leads to Figures 5, 6 and 7 . Figure 5 shows that the optimal compensator makes $\lambda$ insensitive to uncertainty, i.e. the CDF resembles a step function which would result if the system is deterministic. The 


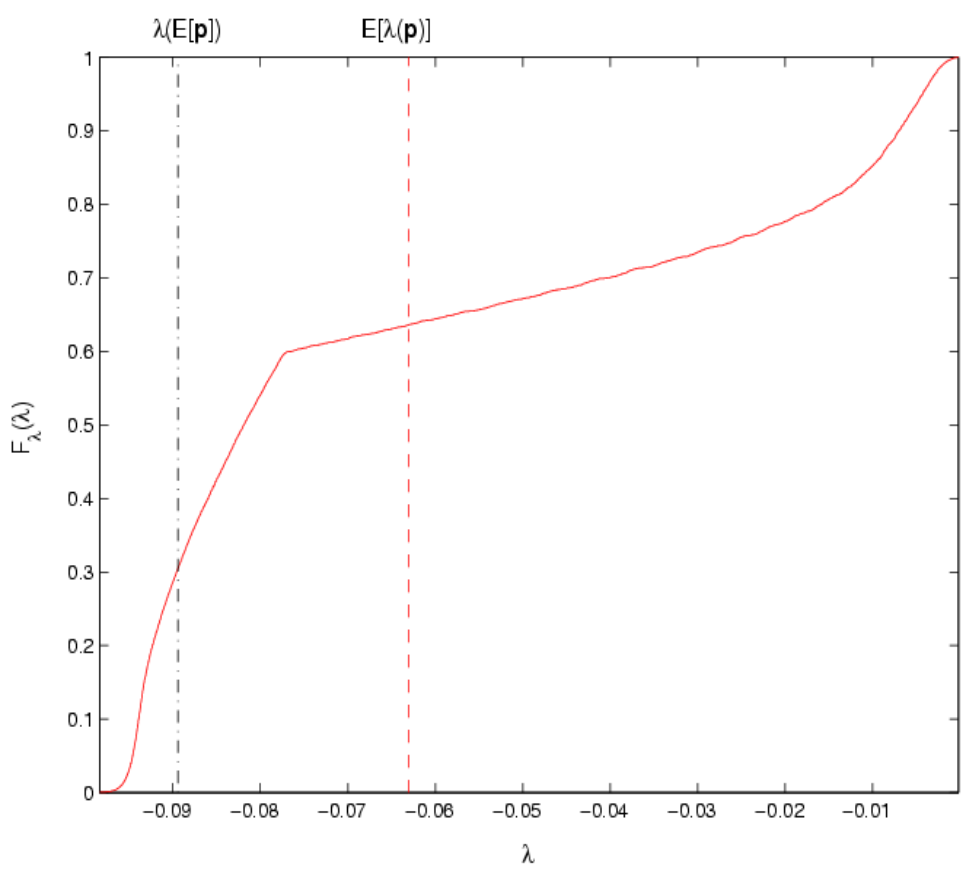

Figure 2. CDF of $\lambda$ for the nominal compensator.

reader must notice that the bound on the stability condition tends to reduce the variability of $\lambda$. This artificially reduces the design space, i.e. there might exist solutions with a better performance in which large variances in $\lambda$ occur. This is a consequence of the form of the bound. This problem has been studied in reference ${ }^{13}$ using reliability metrics exclusively. We will refer to this compensator as the reliability compensator. The comparison between the time responses for the reliability compensator and Figures 6 and 7 show substantial differences between the two solutions. While the control for the reliability based compensator intends to keep the process within the strip $|u|<0.5$, the mean and variance based solution intends to concentrate the random process about the target function $\hat{u}=0$. It can be seen that this is achieved by inducing oscillations about the target function. The reader should also notice that excursions beyond the failure boundaries in a reliability formulation, are not penalized according to the severity of the violation. This is in sharp contrast with the mean and variance formulation. The CPU time for the finer assessment, i.e. the one using $n=500 \mathrm{~m} \mathbf{p}$ samples, is $77 \mathrm{~s}$. For a given number of samples, the estimation of means and variances is in general more accurate than the estimation of failure probabilities. Small 

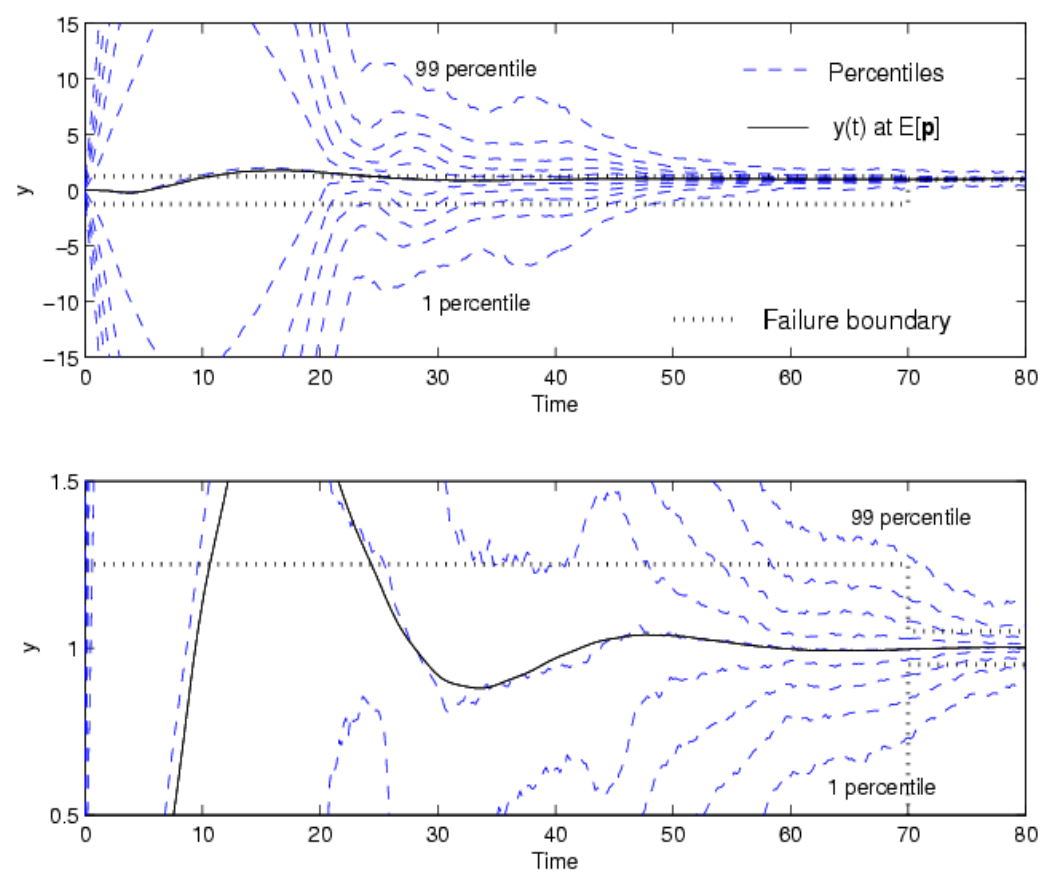

Figure 3. $y(t)$ for the nominal compensator. A zoom is shown below.

sample sets however, result in larger estimation errors of the moments, which could lead to the selection of the wrong conditions in Equations (18-19) and (21-22).

Next, the FSMSO method is used. The first and second order derivatives for all the metrics of interest, i.e. closed-loop poles, output, control and loop transfer function, were derived analytically. Some of the required expressions are as follows

$$
\begin{gathered}
{\left[\begin{array}{c}
\dot{\tilde{\mathbf{x}}} \\
\partial \dot{\tilde{\mathbf{x}}} \\
\partial^{2} \dot{\tilde{\mathbf{x}}}
\end{array}\right]=\left[\begin{array}{ccc}
\tilde{\mathbf{A}} & \mathbf{0} & \mathbf{0} \\
\partial \tilde{\mathbf{A}} & \tilde{\mathbf{A}} & \mathbf{0} \\
\partial^{2} \tilde{\mathbf{A}} & 2 \partial \tilde{\mathbf{A}} & \tilde{\mathbf{A}}
\end{array}\right]\left[\begin{array}{c}
\tilde{\mathbf{x}} \\
\partial \tilde{\mathbf{x}} \\
\partial^{2} \tilde{\mathbf{x}}
\end{array}\right]+\left[\begin{array}{c}
\tilde{\mathbf{B}} \\
\partial \tilde{\mathbf{B}} \\
\partial^{2} \tilde{\mathbf{B}}
\end{array}\right] u} \\
{\left[\begin{array}{c}
\tilde{\mathbf{y}} \\
\partial \tilde{\mathbf{y}} \\
\partial^{2} \tilde{\mathbf{y}}
\end{array}\right]=\left[\begin{array}{ccc}
\tilde{\mathbf{C}} & \mathbf{0} & \mathbf{0} \\
\partial \tilde{\mathbf{C}} & \tilde{\mathbf{C}} & \mathbf{0} \\
\partial^{2} \tilde{\mathbf{C}} & 2 \partial \tilde{\mathbf{C}} & \tilde{\mathbf{C}}
\end{array}\right]\left[\begin{array}{c}
\tilde{\mathbf{x}} \\
\partial \tilde{\mathbf{x}} \\
\partial^{2} \tilde{\mathbf{x}}
\end{array}\right]}
\end{gathered}
$$

where $\tilde{\mathbf{A}}, \tilde{\mathbf{B}}, \tilde{\mathbf{C}}$, and $\tilde{\mathbf{D}}$ were given in Equations (3) and (4), $\tilde{\mathbf{x}}=\left[\mathbf{x}_{c}^{T}, \mathbf{x}^{T}\right]^{T}, \partial[\cdot]$ indicates a 


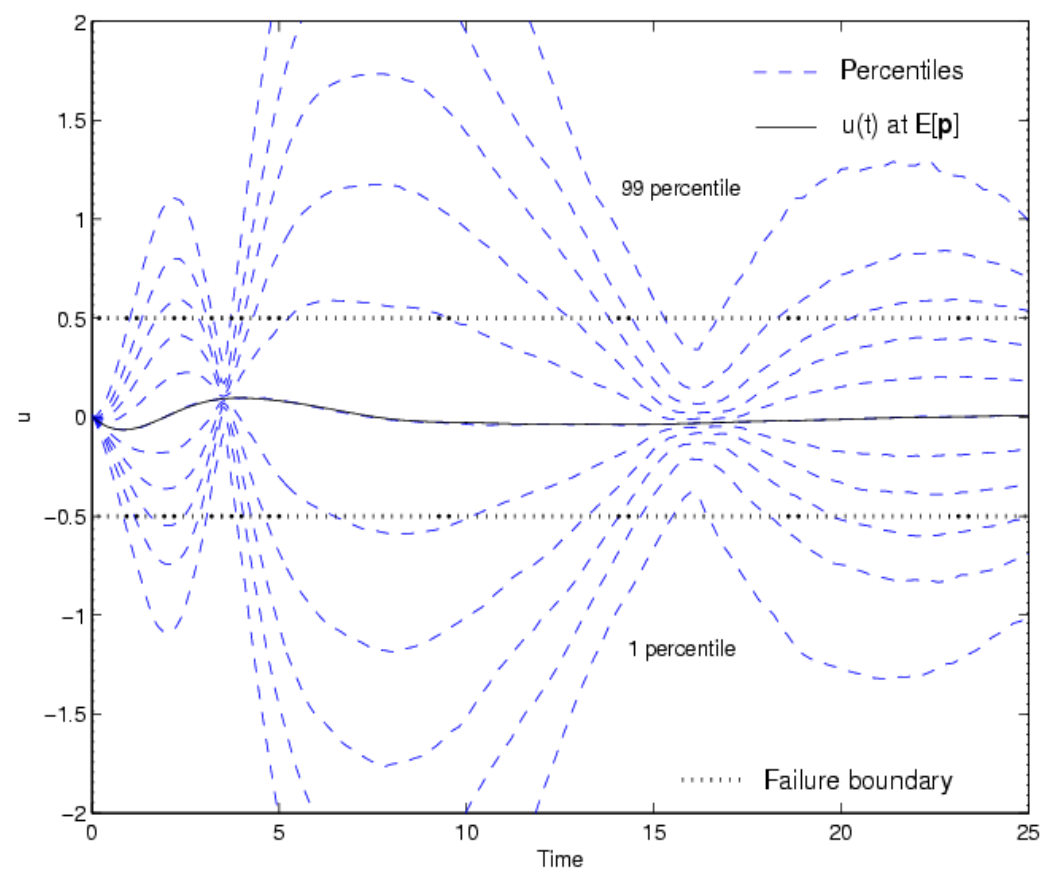

Figure 4. $u(t)$ for the nominal compensator.

derivative with respect to $\mathbf{p}_{(i)}$ excluding initial conditions, and

$$
\begin{aligned}
& \tilde{\mathbf{A}} \triangleq\left[\begin{array}{cc}
\mathbf{A}_{c} & -\mathbf{B}_{c} \mathbf{C} \\
\mathbf{B C}_{c} & \mathbf{A}-\mathbf{B D}_{c} \mathbf{C}
\end{array}\right] \\
& \partial \tilde{\mathbf{A}}=\left[\begin{array}{cc}
\mathbf{0} & -\mathbf{B}_{c} \partial \mathbf{C} \\
\partial \mathbf{B C}_{c} & \partial \mathbf{A}-\mathbf{B D}_{c} \partial \mathbf{C}-\partial \mathbf{B D}_{c} \mathbf{C}
\end{array}\right] \\
& \partial^{2} \tilde{\mathbf{A}}=\left[\begin{array}{cc}
\mathbf{0} & -\mathbf{B}_{c} \partial^{2} \mathbf{C} \\
\partial^{2} \mathbf{B C}_{c} & \partial^{2} \mathbf{A}-\mathbf{B D}_{c} \partial^{2} \mathbf{C}-2 \partial \mathbf{B D}_{c} \partial \mathbf{C}-\partial^{2} \mathbf{B D}_{c} \mathbf{C}
\end{array}\right] \\
& \tilde{\mathbf{B}} \triangleq\left[\begin{array}{ll}
\mathbf{B}_{c} & \mathbf{B D}_{c}
\end{array}\right]^{T}, \partial \tilde{\mathbf{B}}=\left[\begin{array}{ll}
\mathbf{0} & \partial \mathbf{B D}_{c}
\end{array}\right]^{T}, \partial^{2} \tilde{\mathbf{B}}=\left[\begin{array}{ll}
\mathbf{0} & \partial^{2} \mathbf{B D}_{c}
\end{array}\right]^{T},
\end{aligned}
$$




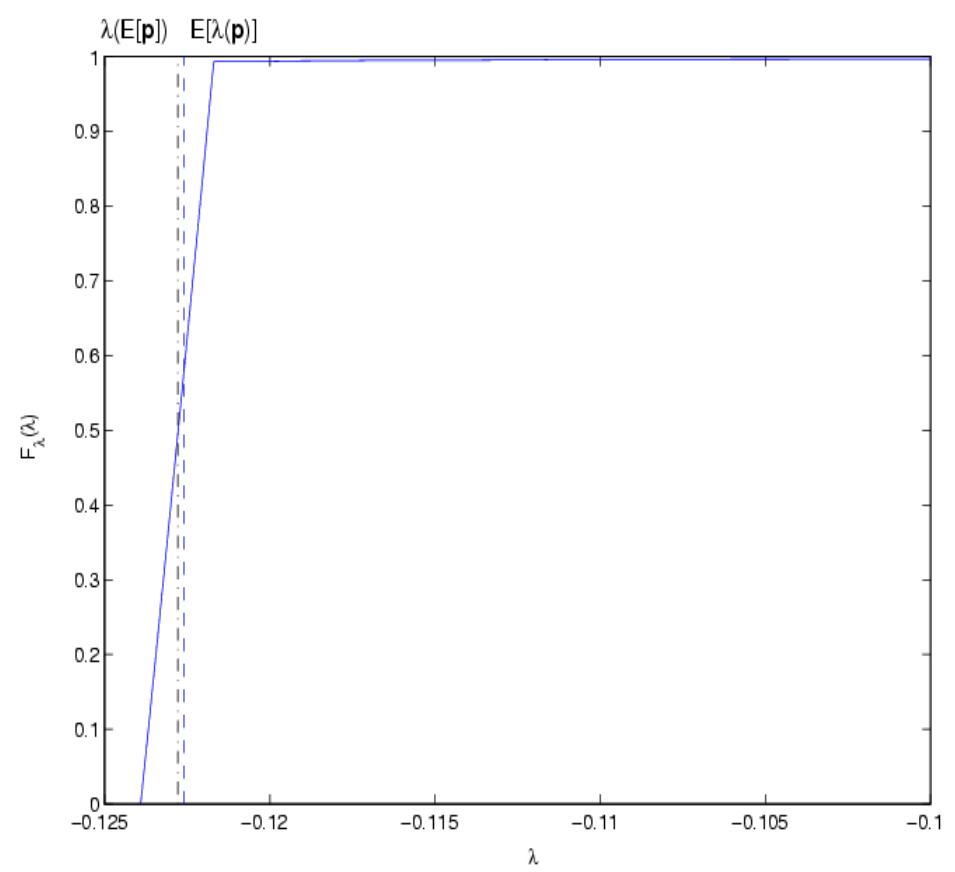

Figure 5. CDF of $\lambda$ for the mean and variance based compensator.

$$
\tilde{\mathbf{C}} \triangleq\left[\begin{array}{ll}
\mathbf{0} & \mathbf{C}
\end{array}\right], \partial \tilde{\mathbf{C}}=\left[\begin{array}{ll}
\mathbf{0} & \partial \mathbf{C}
\end{array}\right], \partial^{2} \tilde{\mathbf{C}}=\left[\begin{array}{ll}
\mathbf{0} & \partial^{2} \mathbf{C}
\end{array}\right]
$$

In these expressions, the subscript $c$ refers to the state space representation of the compensator $K$ while the matrices with no subscript refer to the open-loop plant. The time evolution of the sensitivities is calculated by solving the state space model in Equations (32-33). Sensitivities with respect to initial conditions can be analytically calculated via the matrix exponential. Such developments as well as the ones for the other sensitivities are omitted due to space limitations. In general, the analyses based on FSMSO were inaccurate when compared with sampling. Even though the CPU time per analysis was reduced to 2 seconds, large errors in the estimation precluded its use for synthesis. Table 2 shows a comparison between the assessments resulting from HSS and FSMSO as the variance of the uncertain parameters is increased. The same PDFs for the input uncertainty of Table 1 are used while the means are kept constant. The right most column shows the average relative error in the components of c. For the Beta distributions, the increase of the variance was attained by enlarging the support. The terms resulting from the crossed derivatives were neglected. It can be observed that the accuracy of the FSMSO estimation rapidly decreases 

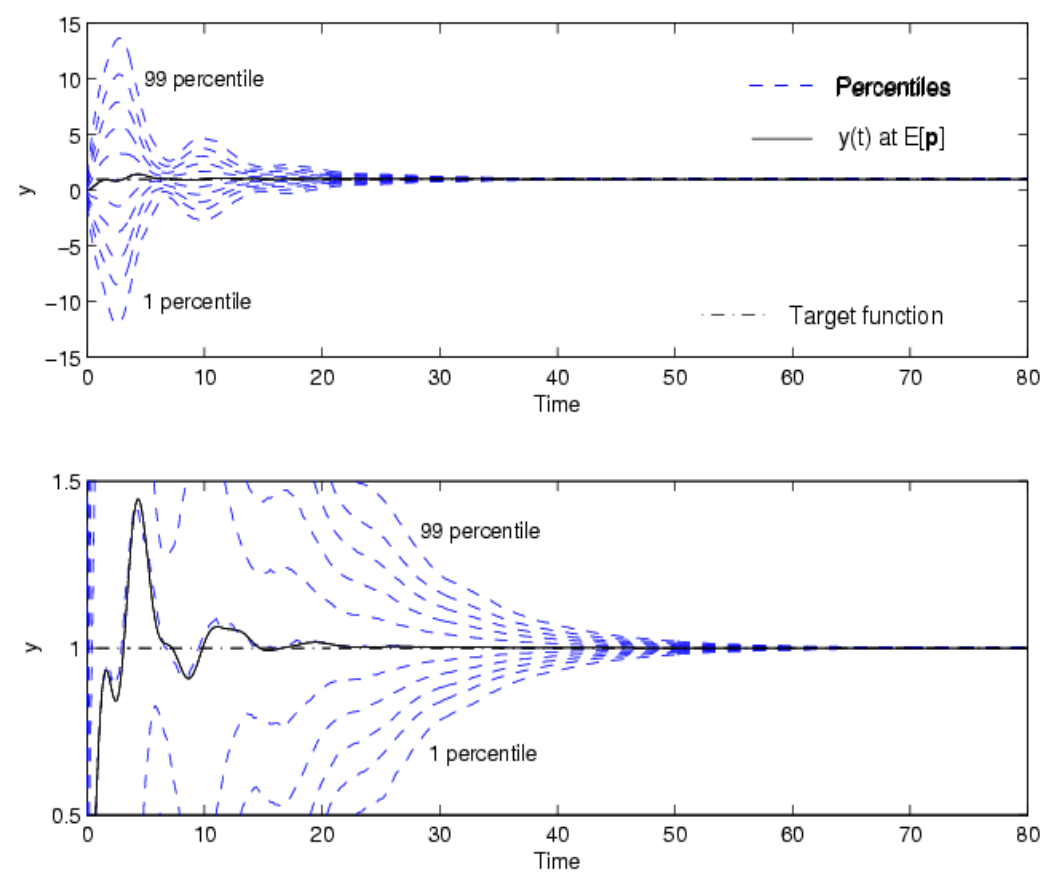

Figure 6. $y(t)$ for the mean and variance based compensator. A zoom is shown below.

as $f_{\mathbf{p}}(\mathbf{p})$ is less concentrated about its mean. This trait is obvious since large excursions from $\mathrm{E}[\mathbf{p}]$ might considerably degrade the accuracy of the Taylor approximation.

The use of reliability bounds introduces unnecessary conservatism into the problem. To show the reduction in the design space caused by using $\bar{b}_{\lambda}(0)$, we have solved this problem using $\bar{r}_{\lambda}(0)$ instead. This practice results in the compensator $\mathbf{k}_{4}=10^{5}[0.9082,0.3590$, $1.1773,0.2372,0.001,0.0616,1.0432,1.196]^{T}$ for which $\mathbf{c}=\left[1.86 \times 10^{-5}, 0.0052,0.0121,1.845 \times\right.$ $\left.10^{-4}, 6.10 \times 10^{-5}\right]^{T}$. Figure 8 shows that $\Delta_{\lambda}$ is about 10 times larger than the one in Figure 5. Actually, $\mathrm{V}[\lambda]$ is about 2276 times larger. Large variance values in a formulation using reliability bounds lead to the penalization of the compensator in spite of its actual improved performance. The reader should also notice that there is an offset of about $10 \%$ between $\mathrm{E}[\lambda]$ and $\lambda(\mathrm{E}[\mathbf{p}])$. Performance improvements in all the metrics can be seen in Figures 9 and 10 . 


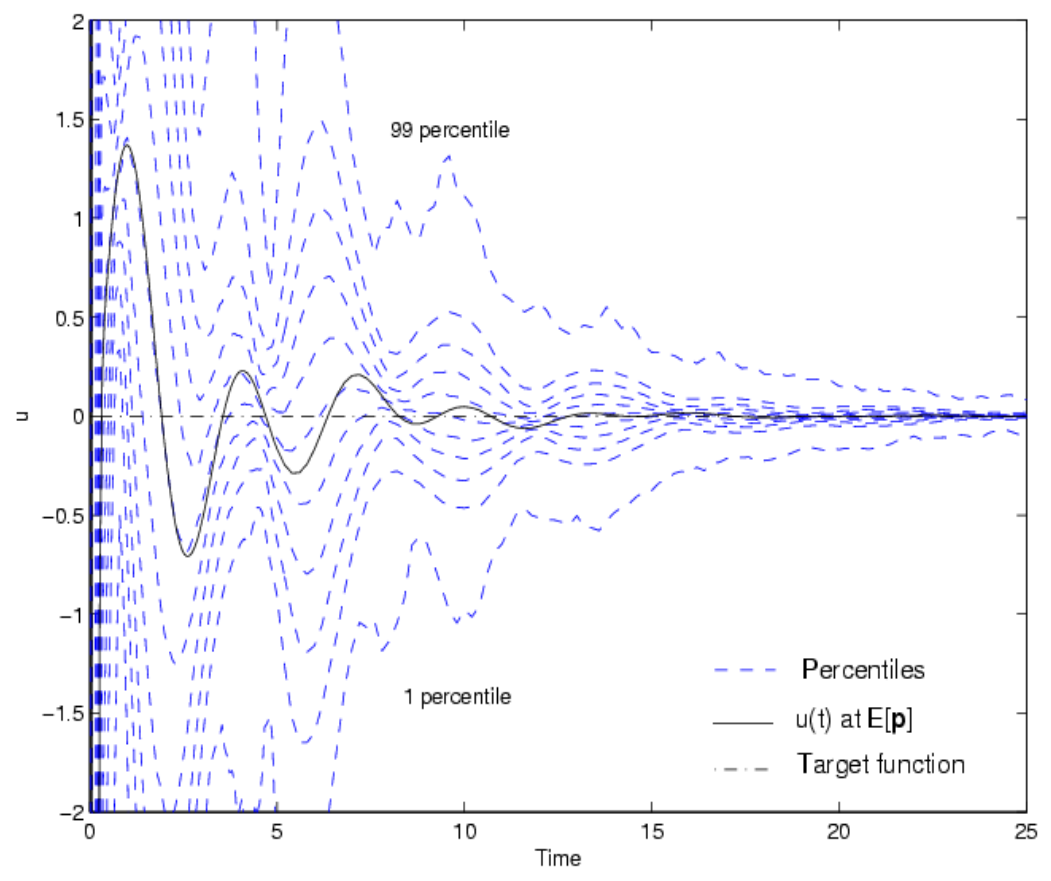

Figure 7. $u(t)$ for the mean and variance base compensator

\section{B. Disturbance Rejection for a Flexible Beam}

The second example will focus on a reliability-based disturbance rejection solution for a flexible beam test article with both physical and modal parameter uncertainties. The system consists of a flexible thin aluminum blade, one-meter long, attached at its base to a hub motor. The hub motor is the control actuator for the system. At the tip of the beam, there is a reaction wheel that serves as a disturbance generator. The test article has nine sensors that may be used in any combination for either feedback or performance output monitoring. The finite element method is used to model this system by utilizing Euler-Bernoulli planar beam elements. A complete description of the flexible beam test article ${ }^{20}$ is available.

For this paper a SISO problem where the input $\mathbf{u}$ is the hub motor torque and the measured output $\mathbf{y}$ is the tip velocity, is studied. The tip reaction wheel disturbance is modeled by passing a Gaussian white noise process through a second-order linear low-pass filter, with parameters $\zeta=0.8$ and $\omega_{n}=200 \pi \mathrm{rad} / \mathrm{s}$. The first five modes of the elastic structure are used to build a state space realization of the plant. This, in addition to the disturbance model leads to an open-loop system where $\mathbf{x} \in \mathbb{R}^{12}, \mathbf{u} \in \mathbb{R}$ and $\mathbf{y} \in \mathbb{R}$. 
Table 2. Comparison of HSS and FSMSO for $\mathrm{k}_{3}$.

\begin{tabular}{|c|c|c|}
\hline $\begin{array}{c}\mathrm{V}\left[\mathbf{p}_{(i)}\right] \text { for } \\
i=1, \ldots 7\end{array}$ & $\begin{array}{c}\text { Cost vector } \\
\mathbf{c}\end{array}$ & $\begin{array}{c}\text { Average } \\
\text { error }\end{array}$ \\
\hline $1 \times 10^{-4}$ & $\mathbf{c}_{H S S}=10^{-3}[0.000823,0.0096,0.5880,0.0058,0.0013]$ & $0.3 \%$ \\
& $\mathbf{c}_{F S M S O}=10^{-3}[0.000827,0.0095,0.5880,0.0058,0.0001]$ & \\
\hline $1 \times 10^{-3}$ & $\mathbf{c}_{H S S}=10^{-3}[0.00824,0.0110,0.6560,0.0580,0.0136]$ & $4 \%$ \\
& $\mathbf{c}_{F S M S O}=10^{-3}[0.00823,0.0108,0.7720,0.0580,0.0133]$ & \\
\hline $3 \times 10^{-3}$ & $\mathbf{c}_{H S S}=10^{-3}[5.1000,0.0505,0.8800,0.1745,0.0446]$ & $57 \%$ \\
& $\mathbf{c}_{F S M S O}=10^{-3}[0.0247,0.0194,1.9160,0.1740,0.0408]$ & \\
\hline $4 \times 10^{-3}$ & $\mathbf{c}_{H S S}=10^{-3}[32.400,0.0013,1.0880,0.2330,0.0626]$ & $454 \%$ \\
& $\mathbf{c}_{F S M S O}=10^{-3}[0.0329,0.0267,2.8680,0.2320,0.0548]$ & \\
\hline
\end{tabular}

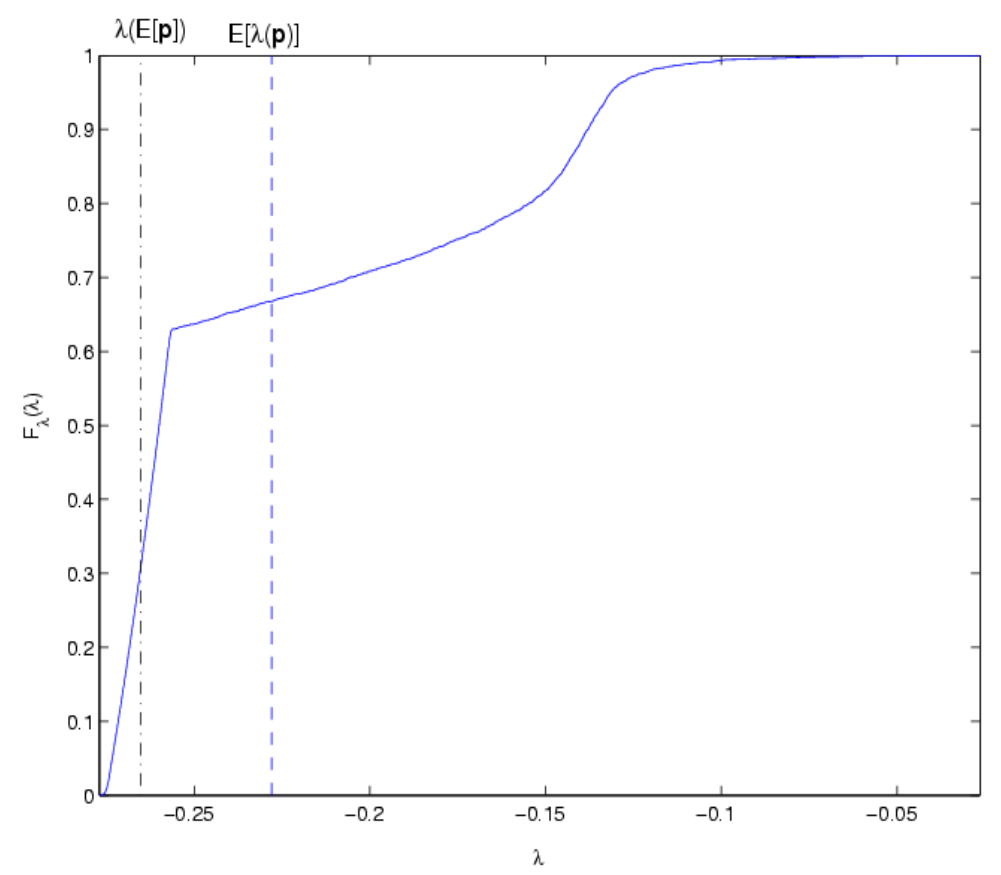

Figure 8. CDF of $\lambda$ for a compensator with parameters $\mathbf{k}_{4}$.

The uncertain parameters are the Young's Modulus $E(\mathrm{~Pa})$, the density $\rho\left(\mathrm{Kg} / \mathrm{m}^{3}\right)$ and the damping ratios of the retained vibration modes. This set leads to $\mathbf{p}=\left[E, \rho, \xi_{1}, \xi_{2}, \xi_{3}, \xi_{4}, \xi_{5}\right]^{T}$, whose components are assumed independent. The corresponding PDFs are given in Table 

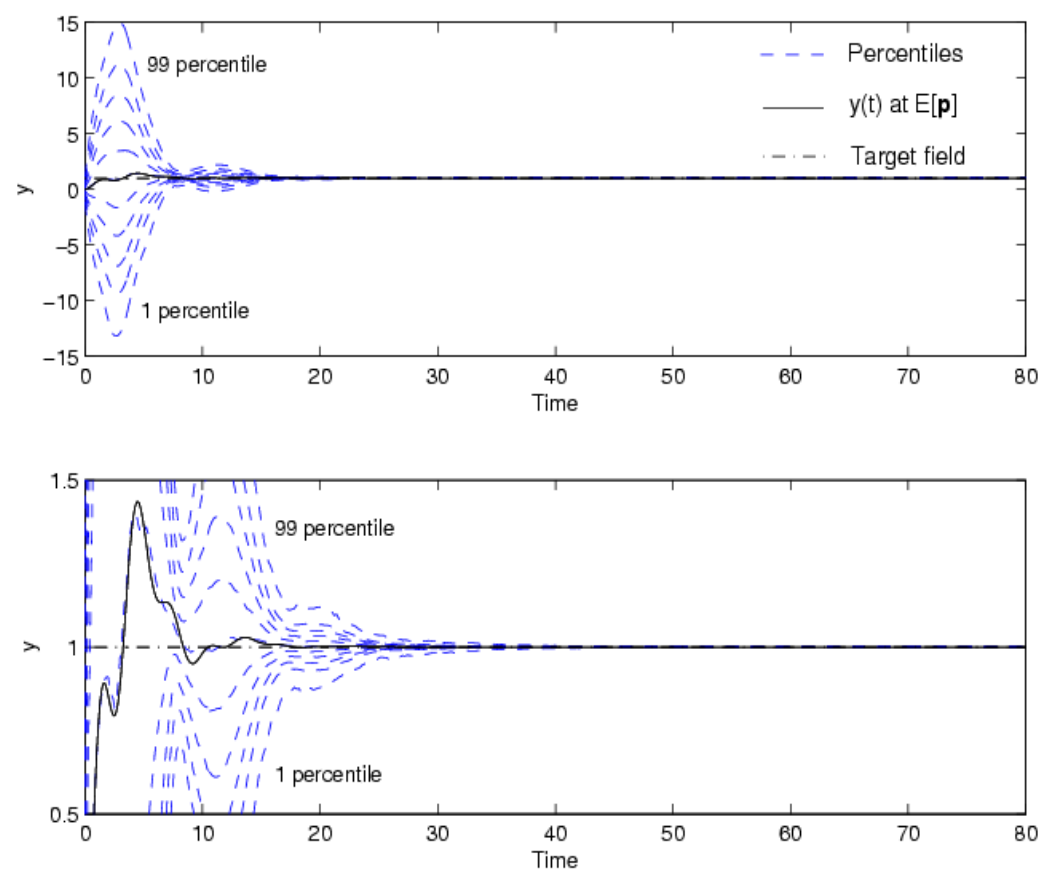

Figure 9. $y(t)$ for a compensator with parameters $\mathbf{k}_{4}$. A zoom is shown below.

3. The mean value of the parameters $E[\mathbf{p}]$ is set to coincide with the parameters in the finite element model. These mean values were chosen to match experimental data, while the supports of the distributions were set according to reasonable ranges of variation. The shapes of the PDFs were arbitrarily set. Performance requirements on stability and the output RMS are considered. Full-state feedback with a full-order observer determine the control structure.

\section{Nominal Compensator}

As before, a baseline compensator for the nominal plant is designed such that the RMS value is minimized. The resulting compensator, denoted as $\mathbf{d}_{1}$, leads to $y_{r m s}=0.011 \mathrm{~m} / \mathrm{s}$. The propagation of the uncertainty prescribed in Table 3 through the closed loop-system show that this compensator is robustly unstable with $\bar{r}_{\lambda}(0)=0.235$. 


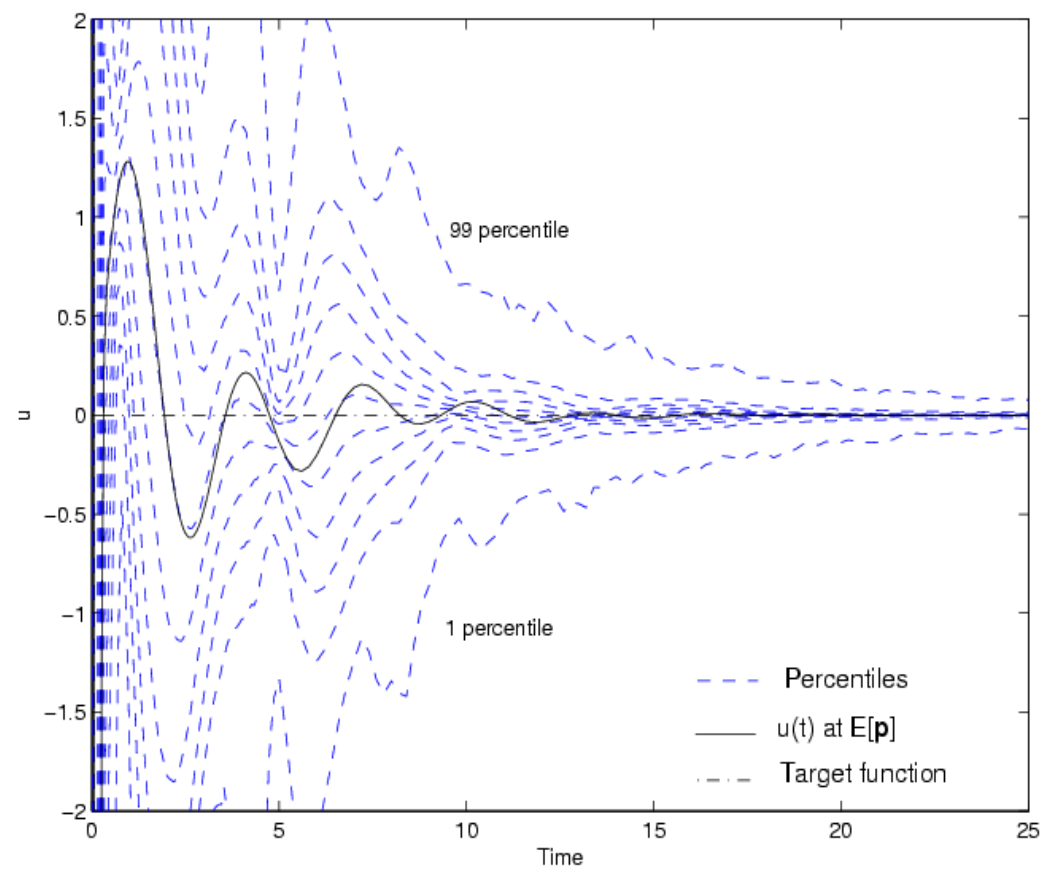

Figure 10. $u(t)$ for a compensator with parameters $\mathbf{k}_{4}$

\section{Reliability-based Compensator}

For the sake of comparison, a reliability compensator will be synthesized using the hybrid method of reference. ${ }^{13}$ In particular, a shapeable failure domain for the RMS requirement is assumed. This leads to the cost vector $\mathbf{c}=\left[\bar{r}_{\lambda}(0), \bar{r}_{y r m s}(\mathbf{e})+\gamma_{y r m s}\right]^{T}$, where $\mathbf{e} \in[0,0.05]$ and $\gamma_{y r m s}=\mathbf{e}$. The selected control structure makes the feedback gain $\mathbf{G}$, the observer gain $\mathbf{L}$, and the RMS failure boundary e, the design variables. Recall that the separation principle does not hold. The resulting closed-loop dynamics is given by Equations (3) and (4). Notice that although the observer is deterministic, all the closed-loop poles are random.

The synthesis approach with $\mathbf{w}=[20,1]^{T}$ leads to a compensator with parameters $\mathbf{d}_{2}$, for which $\bar{r}_{\lambda}(0)=0, \mathbf{e}=0.0139 \mathrm{~m} / \mathrm{s}, \bar{r}_{y r m s}(\mathbf{e})=3.6 \times 10^{-3}$ and $\mathbf{c}=\left[0,3.6 \times 10^{-3}\right]^{T}$. The probabilistic analysis of $\mathbf{d}_{2}$ leads to Figures 11-12. Figure 11 shows that the random variable $y_{r m s}$ is moved toward zero, by virtue of the non-fixed failure boundary. In addition, Figure 12 shows Bode magnitude plots of the disturbance to output transfer function, namely $T_{z y}$. Notice that differences in the low-frequency portion of the diagram have a bigger impact on the RMS value. In addition, considerable variability in the closed-loop Bode magnitude 
Table 3. Uncertainty model.

\begin{tabular}{|c|c|c|}
\hline$E$ & $\Delta_{E}=10^{10}[5.226,7.839]$ & $f_{E}(E)=B(5,5)$ \\
\hline$\rho$ & $\Delta_{\rho}=[2280,3420]$ & $f_{\rho}(\rho)=B(3,3)$ \\
\hline$\xi_{1}$ & $\Delta_{\xi_{1}}=[0.08,0.12]$ & $f_{\xi 1}\left(\xi_{1}\right)=B(2,2)$ \\
\hline$\xi_{2}$ & $\Delta_{\xi_{2}}=[0.0252,0.0378]$ & $f_{\xi 2}\left(\xi_{2}\right)=B(2,2)$ \\
\hline$\xi_{3}$ & $\Delta_{\xi_{3}}=[0.02,0.03]$ & $f_{\xi 3}\left(\xi_{3}\right)=B(2,2)$ \\
\hline$\xi_{4}$ & $\Delta_{\xi_{4}}=[0.0304,0.0456]$ & $f_{\xi 4}\left(\xi_{4}\right)=B(2,2)$ \\
\hline$\xi_{5}$ & $\Delta_{\xi_{3}}=[0.02,0.03]$ & $f_{\xi 5}\left(\xi_{5}\right)=B(2,2)$ \\
\hline
\end{tabular}

plot as well as a significant reduction in the damping of the first mode are attained near 20 $\mathrm{rad} / \mathrm{s}$. It is interesting to notice that even though $\mathbf{d}_{2}$ leads to a robustly stable closed-loop system in Equation (3), the full-state feedback subsystem $\tilde{\mathbf{A}}_{1,1}$ and the full-order observer subsystem $\tilde{\mathbf{A}}_{2,2}$ have a non-zero probability of instability. This indicates that the Separation Principle artificially reduces the design space once uncertainty is present.

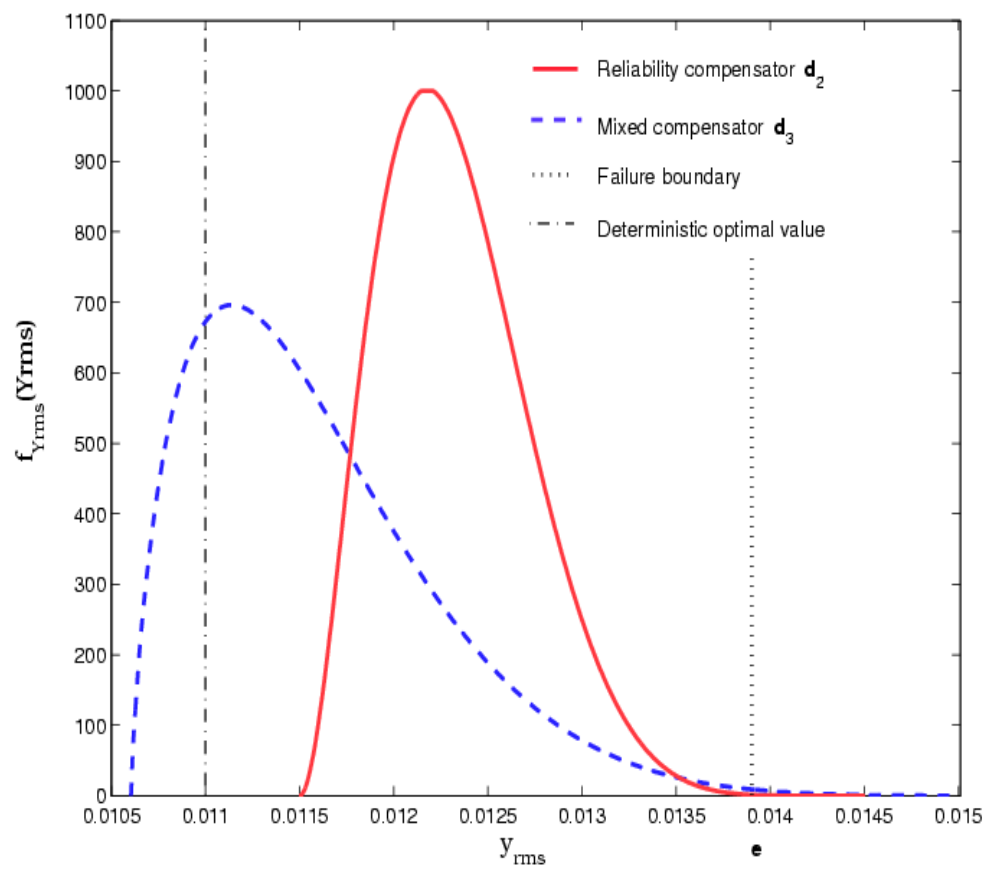

Figure 11. PDFs for the reliability based and the robustness based compensators. 


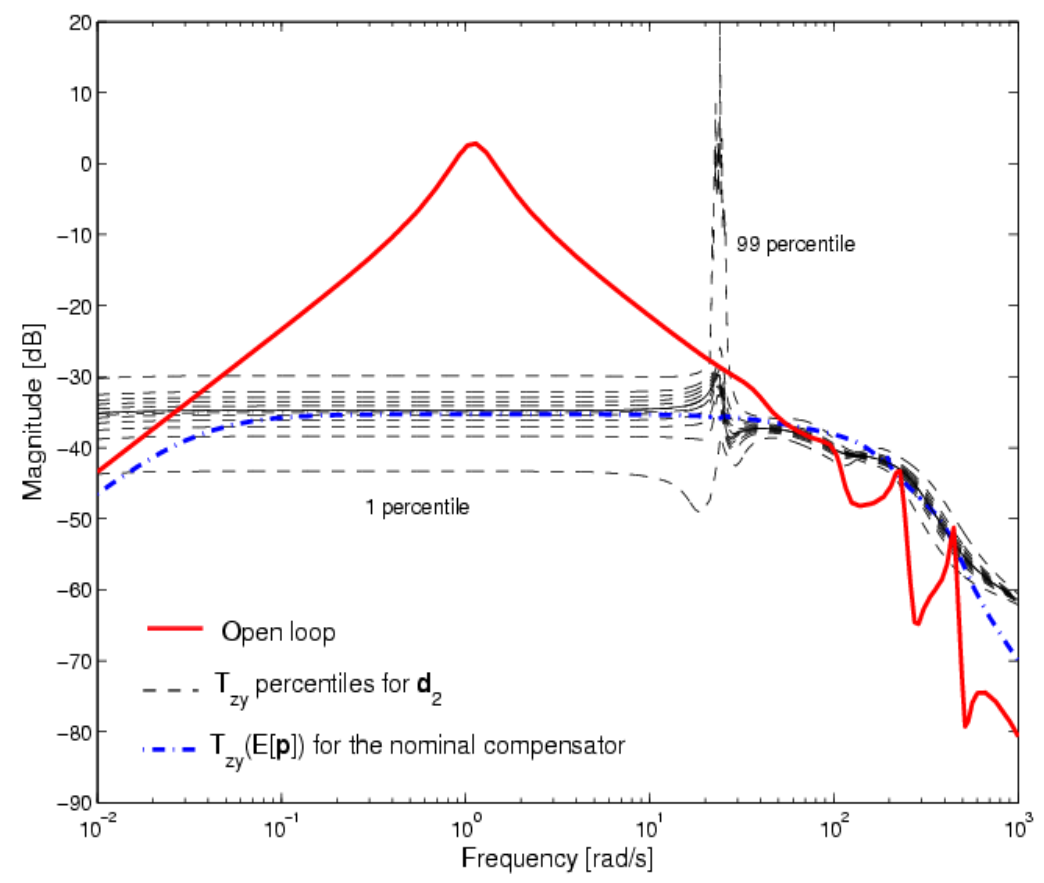

Figure 12. Bode diagrams of $T_{z y}$ for $\mathrm{d}_{2}$.

\section{Mixed Compensator}

Lets take $\mathbf{c}=\left[\bar{r}_{\lambda}(0), \tau_{y r m s}(0)\right]^{T}$, where the hybrid approach of reference ${ }^{13}$ will be used for the stability metric and HSS for the RMS metric. The synthesis algorithm leads to $\mathbf{d}_{3}$, for which $\mathbf{c}=\left[0,1.34 \times 10^{-4}\right]^{T}$. This compensator leads to the dashed line in Figure 11. Comparing both solutions, we see that while the PDF corresponding to the reliability-based compensator has less probability of exceeding the boundary value of $\mathbf{e}=0.0139 \mathrm{~m} / \mathrm{s}$, the robustness-based compensator leads to a PDF which is much more concentrated toward the ideal value of zero. This clearly shows that the conceptual differences between the two formulations. Since there is no conservatism in the selection of the nominal plant, i.e., $G(\mathrm{E}[\mathbf{p}])$ is not the most difficult plant to control, $y_{r m s}=0.011 \mathrm{~m} / \mathrm{s}$ does not necessarily bound the supports of the PDFs. This can be observed in Figure 11, where the support corresponding to $\mathbf{d}_{3}$ contains $y_{r m s}=0.011$ $\mathrm{m} / \mathrm{s}$. 


\section{Mean and Variance based Compensator}

For this case we assume $\mathbf{c}=\left[\bar{b}_{\lambda}(0), \tau_{y r m s}(0)\right]^{T}$, where the FSMSO method will be used to estimate both indexes. In contrast to the satellite attitude example, the accuracy of the FSMSO method made it suitable for synthesis. Analytical sensitivities were used in the moments approximations. For instance, the sensitivities of the closed-loop poles that determine $\lambda$ are given by

$$
\begin{aligned}
\tilde{\mathbf{A}} \mathbf{v}_{j} & =s_{j} \mathbf{v}_{j} \\
\partial s_{j} & =\mathbf{z}_{j}^{T} \partial \tilde{\mathbf{A}} \mathbf{v}_{j} \\
\partial^{2} s_{j} & =\mathbf{z}_{j}^{T}\left[\partial^{2} \tilde{\mathbf{A}}+\left(\partial \tilde{\mathbf{A}}-\partial s_{j} \mathbf{I}\right)\left(s_{j} \mathbf{I}-\tilde{\mathbf{A}}\right)^{-1} \partial \tilde{\mathbf{A}}+\partial \tilde{\mathbf{A}}\left(s_{j} \mathbf{I}-\tilde{\mathbf{A}}\right)^{-1}\left(\partial \tilde{\mathbf{A}}-\partial s_{j} \mathbf{I}\right)\right] \mathbf{v}_{j}
\end{aligned}
$$

where $\mathbf{z}_{j}$ is the $j$ th eigenvector of $\tilde{\mathbf{A}}^{T}$, the right and left eigenvalues are normalized, i.e. $\mathbf{z}_{j}^{T} \mathbf{v}_{j}=1$, and and non-repeated poles are assumed. See the work of Burchett and Costello ${ }^{21}$ for a review on the subject. Derivatives of the output covariance are given by

$$
\begin{gathered}
\partial \tilde{\mathbf{y}}_{r m s}=\left\{\operatorname{diag}\left[\tilde{\mathbf{C}} \partial \mathbf{Q} \tilde{\mathbf{C}}^{T}+2 \partial \tilde{\mathbf{C}} \mathbf{Q} \tilde{\mathbf{C}}^{T}\right]\right\}^{1 / 2} \\
\partial^{2} \tilde{\mathbf{y}}_{r m s}=\left\{\operatorname{diag}\left[\tilde{\mathbf{C}} \partial^{2} \mathbf{Q} \tilde{\mathbf{C}}^{T}+4 \partial \tilde{\mathbf{C}} \partial \mathbf{Q} \tilde{\mathbf{C}}^{T}+2 \partial^{2} \tilde{\mathbf{C}} \mathbf{Q} \tilde{\mathbf{C}}^{T}+2 \partial \tilde{\mathbf{C}} \partial \mathbf{Q} \partial \tilde{\mathbf{C}}^{T}\right]\right\}^{1 / 2}
\end{gathered}
$$

where the derivatives of the state covariance are given by the solution to the set of Lyapunov equations

$$
\begin{gathered}
\tilde{\mathbf{A}} \partial \mathbf{Q}+\partial \mathbf{Q} \tilde{\mathbf{A}}^{T}+\partial \tilde{\mathbf{A}} \mathbf{Q}+\mathbf{Q} \partial \tilde{\mathbf{A}}^{T}+\partial \tilde{\mathbf{B}} \mathbf{S} \tilde{\mathbf{B}}^{T}+\tilde{\mathbf{B}} \mathbf{S} \partial \tilde{\mathbf{B}}^{T}=0 \\
\tilde{\mathbf{A}} \partial^{2} \mathbf{Q}+\partial^{2} \mathbf{Q} \tilde{\mathbf{A}}^{T}+2 \partial \tilde{\mathbf{A}} \partial \mathbf{Q}+2 \partial \mathbf{Q} \partial \tilde{\mathbf{A}}^{T}+\partial^{2} \tilde{\mathbf{A}} \partial \mathbf{Q}+ \\
\partial \mathbf{Q} \partial^{2} \tilde{\mathbf{A}}^{T}+2 \partial \tilde{\mathbf{B}} \mathbf{S} \partial \tilde{\mathbf{B}}^{T}+\partial^{2} \tilde{\mathbf{B}} \mathbf{S} \tilde{\mathbf{B}}^{T}+\tilde{\mathbf{B}} \mathbf{S} \partial^{2} \tilde{\mathbf{B}}^{T}=0
\end{gathered}
$$

The synthesis algorithm leads to $\mathbf{d}_{4}$, for which $\mathbf{c}=\left[5.33 \times 10^{-6}, 1.33 \times 10^{-4}\right]^{T}$. The resulting $\mathrm{PDF}$ for the RMS is indistinguishable from the one shown in Figure 11 even though the compensators are different. The CDFs of $\lambda$ for $\mathbf{d}_{3}$ and $\mathbf{d}_{4}$ are superimposed in Figure 13. As before, the tendency of the formulation of making $\lambda$ as deterministic as possible is 
apparent. Notice however, that the CPU time required for the analysis of $\mathbf{d}_{3}$ is $421 \mathrm{~s}$ while the one for $\mathbf{d}_{4}$ is $1.22 \mathrm{~s}$. On the other hand, the analysis of $\mathbf{d}_{4}$ via HSS takes $64 \mathrm{~s}$. This exemplifies substantial savings in CPU time which result from using the FSMSO method. In this example, those savings justify the labor required to compute analytical derivatives. The reader must recall, however, that the same method led to inaccurate estimations for the satellite example.

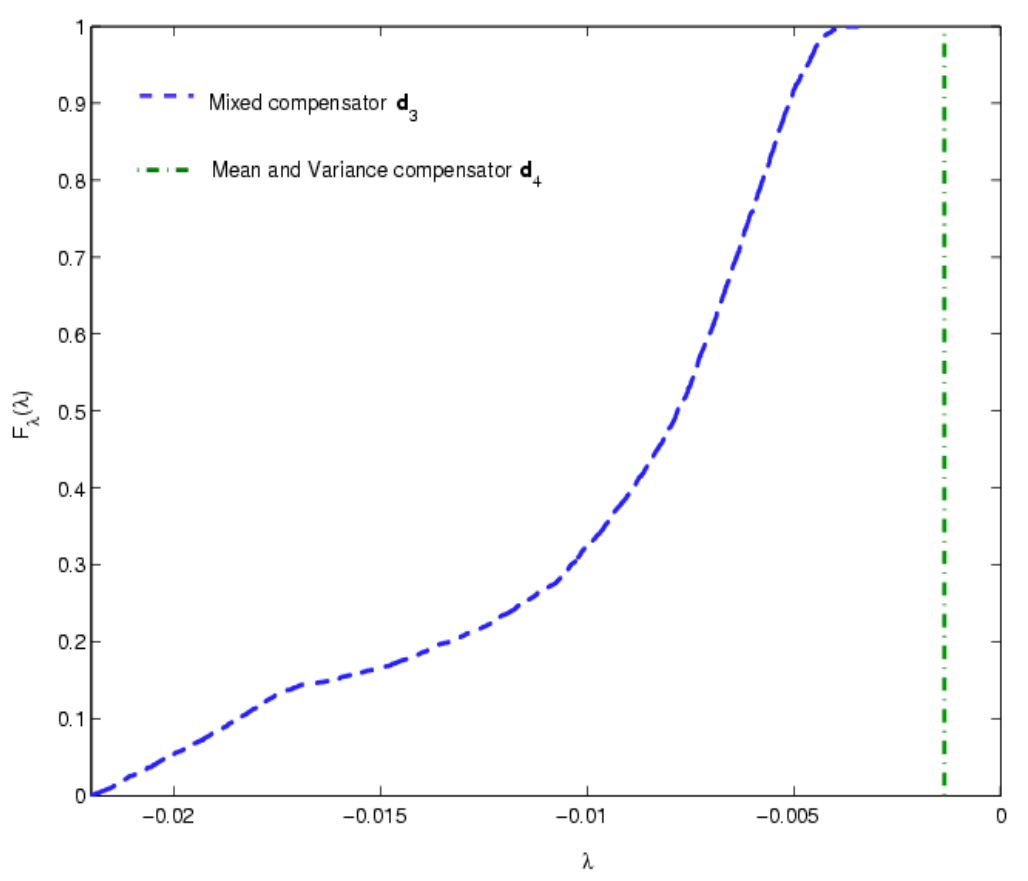

Figure 13. CDFs of $\lambda$ for $d_{3}$ and $d_{4}$.

\section{Conclusions}

This paper proposes a control synthesis methodology for systems with probabilistic uncertainty. Synthesis is performed by solving a multi-objective optimization problem which combines requirements of stability and performance in time- and frequency-domains. In this study, reliability- and robustness- based formulations are proposed and several numerical methods for estimation are examined. In a reliability formulation, the probability of violating design requirements is minimized while admissible domains are contracted toward regions with an improved performance. In a robustness-based formulation, a random metric that 
measures the concentration of the random variable/process about a target scalar/function is minimized. These two formulations lead to compensators with distinctive characteristics. In addition, metrics that bound the reliability metrics proposed, whose estimation only requires of means and variances, are also derived and used for control design.

Some of the fundamental differences between the proposed strategy and conventional robust control methods are: (i) unnecessary conservatism is eliminated since there is not need for convex supports/sets, (ii) the most likely plants are favored during synthesis allowing for probabilistic robust optimality, (iii) the trade off between robust stability and robust performance can be explored numerically, (iv) the uncertainty set, which could be unbounded, is closely related to parameters with clear physical meaning and (v) compensators with improved robust characteristics for a given control structure can be designed, e.g. one can search for a PID controller with best robust characteristics. Examples of attitude control of a simple satellite model and disturbance rejection of a flexible beam are used to elucidate the nature of the problem at hand and to demonstrate the methodology.

\section{References}

${ }^{1}$ Crespo, L. G., "Optimal performance, robustness and reliability based designs of systems with structured uncertainty," Proceedings of American Control Conference, Vol. 5, Denver, CO USA, June 2003, pp. 4219-4224.

${ }^{2}$ Laughlin, D. L., Jordan, K. G., and Morari, M., "Internal model control and process uncertainty mapping uncertainty regions for SISO controller design," International Journal of Control, Vol. 44, No. 6, December 1986, pp. 1675-1698.

${ }^{3}$ Weinmann, A., Uncertain Models and Robust Control, Springer-Verlag, New York, NY USA, 1991.

${ }^{4}$ Zhou, K. and Doyle, J. C., Essentials of Robust Control, Prentice Hall, Upper saddle, New Jersey, 1998.

${ }^{5}$ Marrison, C. and Stengel, R., "Design of Robust Control Systems for a Hypersonic Aircraft," Journal of Guidance, Control, and Dynamics, Vol. 21, January-February 1998, pp. 58-63.

${ }^{6}$ Wang, Q. and Stengel, R. F., "Robust Nonlinear Control of a Hypersonic Aircraft," Journal of Guidance, Control, and Dynamics, Vol. 23, No. 4, July-August 2000, pp. 577-585.

${ }^{7}$ Wang, Q. and Stengel, R. F., "Searching for Robust Minimal-Order Compensators," Journal of Dynamic Systems, Measurement and Control, Vol. 123, June 2001, pp. 233-236.

${ }^{8}$ Calafiore, G., Dabbene, F., and Tempo, R., "Randomized Algorithms for Probabilistic Robustness with Real and Complex Structured Uncertainty," IEEE Transactions on Automatic Control, Vol. 45, No. 12, December 2000, pp. 2218-2235. 
${ }^{9}$ Polyak, B. and Tempo, R., "Probabilistic robust design with linear quadratic regulators," Systems and Control Letters, Vol. 43, 2001, pp. 343-353.

${ }^{10}$ Lagoa, C. M., Li, X., and Sznaier, M., "On the Design of Robust Controllers for Arbitrary Uncertainty Structures," IEEE Transactions on Automatic Control, Vol. 48, No. 11, November 2003, pp. 2061-2065.

${ }^{11}$ Spencer, B. F., Sain, M. K., Won, C.-H., Kaspari, D. C., and Sain, P. M., "Reliability-based measures of structural control robustness," Structural Safety, Vol. 15, 1994, pp. 111-129.

${ }^{12}$ Rackwitz, R., "Reliability analysis, a review and some perspectives," Structural Safety, Vol. 23, 2001, pp. 365-395.

${ }^{13}$ Crespo, L. G. and Kenny, S. P., "Reliability-based control design for uncertain systems," AIAA Journal of Guidance, Control, and Dynamics, Vol. 28, No. 4, 2005.

${ }^{14}$ Wang, Q. and Stengel, R. F., "Robust control of nonlinear systems with parametric uncertainty," Automatica, Vol. 38, 2002, pp. 1591-1599.

${ }^{15}$ Crespo, L. G., "Probabilistic formulations to robust optimal control," 45th AIAA Structures, Structural Dynamics and Materials Conference, Palm Springs, CA USA, April 2005, pp. 1-21, AIAA Paper No. 20041667.

${ }^{16}$ Skogestad, S. and Postlethwaite, I., Multivariable feedback control, John Wiley and Sons, Chichester, England, 1996.

${ }^{17}$ Hokayem, P., Abdallah, C., and Dorato, P., "Quasi-Monte Carlo Methods in Robust Control Design," IEEE Conference on Decision and Control, Maui, HA USA, December 2003, pp. 2435-2440.

${ }^{18}$ Kalagnanam, J. R. and Diwekar, U. M., "An Efficient Sampling Technique for Off-line Quality Control," Technometrics, Vol. 39, No. 3, August 1997, pp. 308-319.

${ }^{19}$ Diwekar, U. M. and Kalagnanam, J. R., "Efficient Sampling Technique for Optimization under Uncertainty," American Institute of Chemical Engineering Journal, Vol. 43, No. 2, February 1997, pp. 440-447.

${ }^{20}$ Kenny, S. P., Optimal rejection of nonstationary narrowband disturbances for flexible systems, Ph.D. thesis, Massachusetts Institute of Technology, Cambridge, MA USA, February, 2002.

${ }^{21}$ Burchett, B. and Costello, M., "QR-Based Algorithm for Eigenvalue Derivatives," AIAA Journal, Vol. 11, No. 40, November 2002, pp. 2319-2322. 